WHOI-88-8

\title{
Cesium and Strontium Isotopes in the Northwestern North Atlantic and Arctic Ocean, 1981-1985
}

by

\author{
Susan A. Casso \\ William R. Clarke \\ Hugh D. Livingston \\ Julianne M. Palmieri \\ and \\ Lolita D. Surprenant
}

Woods Hole Oceanographic Institution
Woods Hole, Massachusetts 02543

April 1988

\section{Technical Report}

Funding was provided by the National Science Foundation under grant Number OCE-8402849

Reproduction in whole or in part is permitted for any purpose of the United States Government. This report should be cited as:

Woods Hole Oceanog. Inst. Tech. Rept., WHOI-88-8.

Approved for publication; distribution unlimited.

Approved for Distribution:

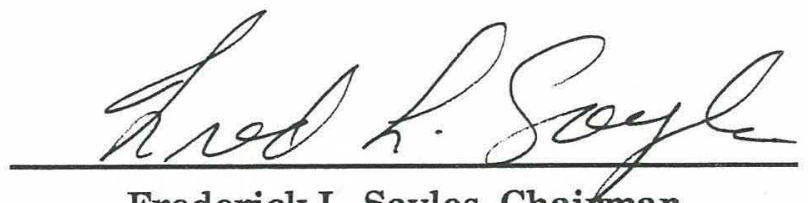

Frederick L. Sayles, Chairman

Department of Chemistry 



\section{TABLE OF CONTENTS}

ABSTRACT. . . . . . . . . . . . . . . . . . . . . 1

INTRODUCTION. . . . . . . . . . . . . . . . . . . . . . . . . . . 1

ACKNOWLEDGEMENTS. . . . . . . . . . . . . . . . . . . . . . . . . 2

R/V METEOR CRUISE 52. . . . . . . . . . . . . . . . . . . . . . . 3-6

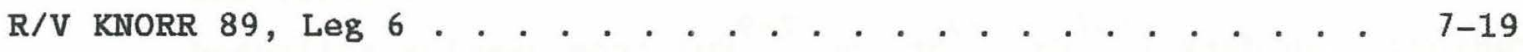

R/V HUDSON WINTER CRUISE TO THE GREENLAND SEA, 1982 . . . . . . . 21-23

ARKTIS II-3: R/V POLARSTERN 1984 CRUISE IN NORTHERN FRAM STRAIT. 25-33

R/V MICHAEL SAARS: FALL 1985 CRUISE TO NORTHEAST BARENTS SEA . . 35-41

ARCTIC ICE-STATIONS: 1979-1985 . . . . . . . . . . . . . . . . 43-47 
ABSTRACT

This report is a follow-up to Woods Hole Oceanographic Institution Technical Report WHOI-84-40. It contains ${ }^{137} \mathrm{Cs}$ and ${ }^{90} \mathrm{Sr}$ data from seawater samples collected on four cruises in the northwestern North Atlantic, Arctic Ocean, and Barents Sea during 1981 to 1985, and radionuclide data $\left({ }^{137} \mathrm{Cs},{ }^{90} \mathrm{Sr}\right.$ and $\left.{ }^{239} \mathrm{Pu}\right)$ from samples collected on a cruise to the Norwegian-Greenland Seas in 1979. Also included are data from four ice stations in the Arctic from 1979 to 1985 . The sample collections were made possible through collaborative efforts with several laboratories. The radionuclide analyses were done at WHOI. 


\section{INTRODUCTION}

The data in this report represent an extension to those which appeared in WHOI-84-40, "Radiocesium and Other Nuclides in the NorwegianGreenland Seas 1981-1982." In that report we have detailed background information on the input to the Arctic Ocean and use of $\mathrm{Cs}$ and $\mathrm{Sr}$ isotopes as circulation tracers. Also in that report we give information on radiochemical methods employed and the format of the results presentation.

Basically this report extends the data in WHOI-84-40 into the Arctic Ocean north of Fram Strait, into the Labrador Sea south of Denmark Strait, into the northeast Barents Sea, and completes the Norwegian/ Greenland Sea data from the 1982 winter cruise by the R/V HUDSON. The data are presented in sections for each cruise or ice-station. A short summary of each field program precedes the relevant data sets. 


\section{ACKNOWLEDGEMENTS}

A great deal of help was provided by many individuals and institutions in the collection of the samples for which data are presented in this report. We appreciate all this help and recognize the generous spirit of cooperation that it represents.

The R/V METEOR 52 samples were collected on this cruise for us by

Dr. Peter Koltermann of the Deutsches Hydrographisches Institut in Hamburg, FRG. The R/V KNORR 89, Leg 6 samples were part of the Transient Tracers in Oceanography North Atlantic Study. Drs. J. H. Swift and V. E. Lee were instrumental in obtaining the R/V HUDSON samples. We collected the $1984 \mathrm{R} / \mathrm{V}$ POLARSTERN samples on the ARKTIS II/3 cruise organized by the Alfred-Wegener-Institute for Polar and Marine Research, Bremerhaven, FRG. Dr. Lars Midttun of the Institute of Marine Research, Bergen, Norway, kindly arranged for the collection of water samples from the eastern Barents Sea on a regular cruise of the Norwegian Fisheries' $R / V$ MICHAEL SAARS.

The samples from the LOREX and CESAR ice-stations were collected through the good offices of Dr. Robert Moore of Dalhousie University, Halifax, N.S., Canada. The FRAM-4 samples were obtained through the efforts of Drs. P. J. Stern and A. Baggeroer of MIT. The AIWEX ice-camp samples were collected by G. C. Anderson of Scripps Institution of Oceanography through arrangements made there by Dr. J. H. Swift.

We thank Marilyn R. Hess for her excellent secretarial assistance throughout this project and in the production of this report. Support for the program came from the U.S. National Science Foundation under grant OCE-8402849 and is acknowledged with thanks. 


\section{R/V METEOR CRUISE 52}

This cruise occupied stations in the Norwegian and Greenland Seas in 1979. Several stations were at locations occupied in 1972 during the GEOSECS expedition and again in 1981 during the Transient Tracers in Oceanography North Atlantic Study. 


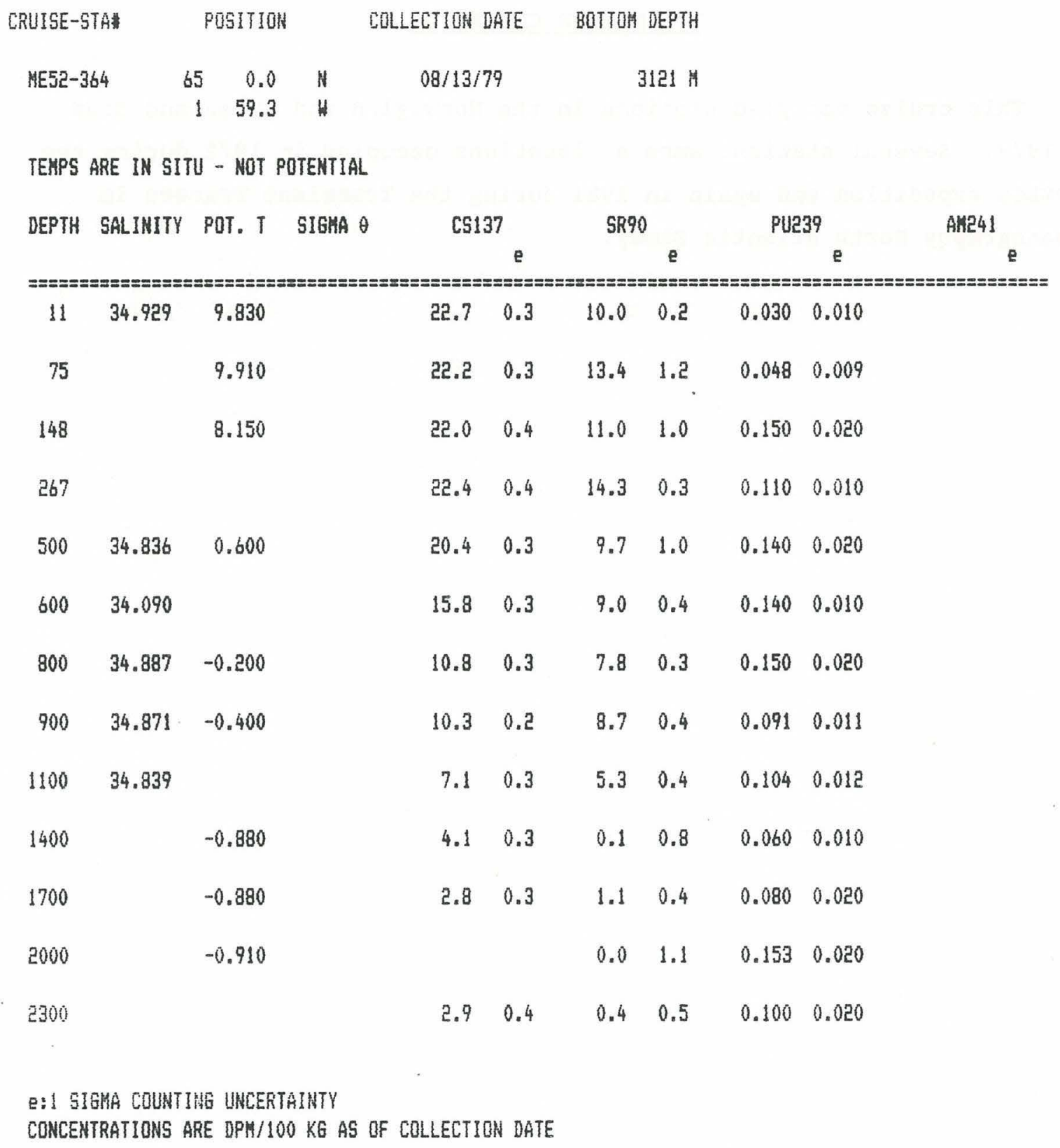




$\begin{array}{rrrrrr}\text { CRUISE-STA } & \text { POSITION } & & \text { COLLECTION DATE } & \text { BOTTOM DEFTH } \\ \text { MESE-367 } & 69 & 50.0 & \text { N } & 08 / 15 / 79 & 3090 \mathrm{H} \\ & 1 & 19.1 & \text { H } & & \end{array}$

CS AND SR DOB CORRECTED; TEMPS ARE IN SITU

\begin{tabular}{|c|c|c|c|c|c|c|c|c|c|c|}
\hline \multirow{2}{*}{ DEPTH } & \multirow[t]{2}{*}{ SALINITY } & \multirow[t]{2}{*}{ POT. T } & \multirow[t]{2}{*}{ SIGKA $\theta$} & \multicolumn{2}{|c|}{ CS137 } & \multicolumn{2}{|c|}{ SR90 } & \multicolumn{2}{|c|}{ Pue39 } & AH241 \\
\hline & & & & & e & & e & & e & e \\
\hline$=x= \pm=x$ & $=x==x=x= \pm=$ & $:== \pm===x=$ & $=x= \pm= \pm= \pm=1$ & $== \pm= \pm=$ & $== \pm=x$ & $== \pm= \pm=$ & $==x=$ & $== \pm==x=$ & $==x== \pm=$ & 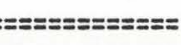 \\
\hline 0 & 35.015 & 8.060 & & 44.9 & 0.4 & 18.4 & 0.5 & 0.090 & 0.020 & \\
\hline 75 & 35.055 & 5.390 & & 54.7 & 0.6 & 19.0 & 0.5 & 0.150 & 0.020 & \\
\hline 150 & 35.019 & 4.420 & & 64.4 & 0.4 & 20.1 & 0.8 & 0.180 & 0.020 & \\
\hline 300 & 35.001 & 3.770 & & 48.0 & 0.6 & 17.0 & 0.8 & 0.190 & 0.020 & \\
\hline 500 & 34.994 & 3.330 & & 42.1 & 0.3 & 18.7 & 0.7 & 0.150 & 0.020 & \\
\hline 600 & 34.966 & 2.920 & & 39.2 & 0.6 & 17.5 & 0.3 & 0.220 & 0.020 & \\
\hline 800 & 34.915 & 0.510 & & 19.6 & 0.3 & 11.1 & 0.4 & 0.200 & 0.020 & \\
\hline 900 & & 0.220 & & 16.8 & 0.5 & 9.6 & 1.0 & 0.180 & 0.020 & \\
\hline 1100 & 34.899 & -0.300 & & 11.4 & 0.3 & 7.4 & 0.4 & 0.160 & 0.020 & \\
\hline 1400 & & & & 5.0 & 0.7 & 3.1 & 0.6 & 0.090 & 0.020 & \\
\hline 1700 & 34.921 & -0.800 & & 2.9 & 0.6 & 1.8 & 0.4 & 0.120 & 0.030 & \\
\hline 2000 & 34.921 & -0.900 & & 2.9 & 0.8 & 3.3 & 0.6 & 0.045 & 0.015 & \\
\hline 2300 & 34.913 & & & 3.5 & 0.2 & 3.6 & 0.2 & 0.060 & 0.020 & \\
\hline 2600 & 34.915 & -0.920 & & & & 1.3 & 0.5 & 0.110 & 0.010 & \\
\hline 2800 & 34.917 & -0.920 & & 3.2 & 0.6 & 1.4 & 0.4 & 0.090 & 0.020 & \\
\hline 3000 & 34.915 & -0.920 & & 3.4 & 0.5 & 1.5 & 0.3 & 0.090 & 0.020 & \\
\hline
\end{tabular}




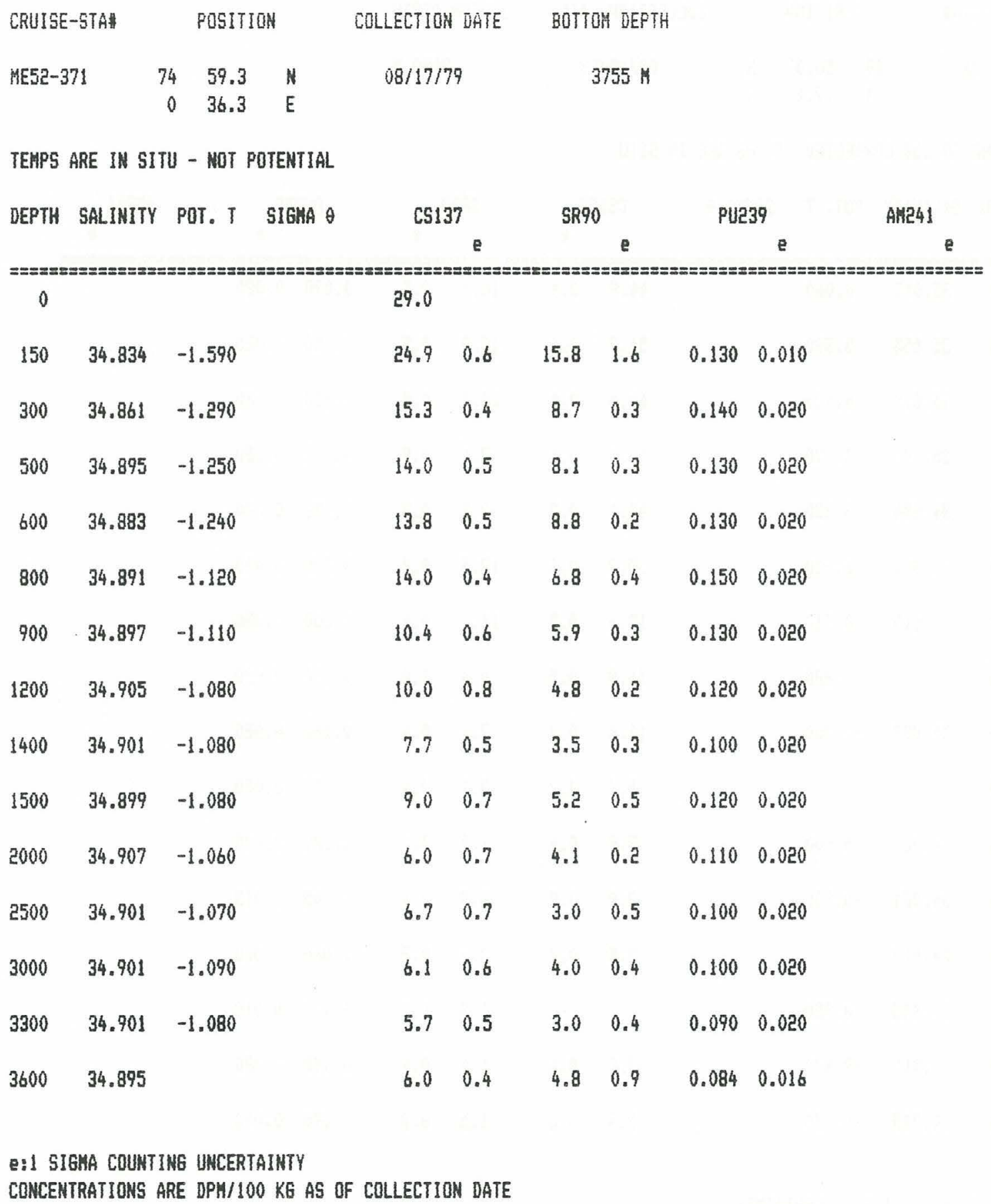


$\underline{R / V ~ K N O R R ~ 89, ~ L e g ~} 6$

Data in this section come from samples from stations occupied on Leg 6 of the Transient Tracers in Oceanography (North Atlantic Study) expedition. This leg occupied stations south of Denmark strait and in the Labrador Sea. The southward advection path of water masses forming North Atlantic Deep Water were a major focus of the sampling strategy. 


$\begin{array}{llcccr}\text { CRUISE-STA } & \text { POSITION } & \text { COLLECTION DATE } & \text { BOTTOH DEPTH } \\ \text { KN89-177 } & 58 & 40.0 & \mathrm{~N} & 08 / 26 / 81 & 3174 \mathrm{M} \\ & 38 & 15.0 & \mathrm{H} & & \end{array}$

\begin{tabular}{|c|c|c|c|c|c|c|c|c|c|}
\hline $\begin{array}{c}\text { DEPTH } \\
\text { H }\end{array}$ & SALINITY & POT. T & SIGMA $\theta$ & & ${ }^{37}$ & SR- & ${ }^{0}$ & CSI & SR \\
\hline & $:= \pm= \pm=x=0$ & $=2 x=x=0$ & $=2= \pm=8=-2$ & $== \pm=x$ & $==2=$ & $=z== \pm=$ & $= \pm= \pm=$ & $== \pm= \pm$ & $== \pm=x$ \\
\hline 0 & & & & 17.7 & 3.4 & & & & \\
\hline 9 & 34.573 & 8.250 & 26.901 & 16.0 & 0.4 & 9.3 & 0.8 & 1.72 & 0.15 \\
\hline 300 & 34.782 & 3.070 & 27.705 & 14.5 & 0.2 & 10.1 & 0.2 & 1.44 & 0.03 \\
\hline 498 & 34.809 & 3.200 & 27.714 & 15.1 & 0.1 & & & & \\
\hline 597 & 34.817 & 3.200 & 27.720 & & & & & & \\
\hline 747 & 34.824 & 3.210 & 27.725 & 21.6 & 2.0 & & & & \\
\hline 996 & 34.841 & 3.253 & 27.734 & 13.3 & 0.2 & 9.4 & 0.5 & 1.41 & 0.08 \\
\hline 1510 & 34.886 & 3.285 & 27.767 & 14.9 & 0.1 & & & & \\
\hline 2106 & 34.950 & 3.188 & 27.827 & 8.3 & 0.3 & 4.5 & 0.4 & 1.84 & 0.18 \\
\hline 2403 & 34.949 & 2.927 & 27.851 & 9.8 & 0.1 & & & & \\
\hline 2905 & 34.934 & 2.450 & 27.881 & 9.1 & 0.2 & 4.5 & 0.5 & 2.02 & 0.23 \\
\hline 3076 & 34.893 & & & 20.0 & 0.1 & & & & \\
\hline 3126 & 34.885 & 1.355 & 27.928 & 18.2 & 0.5 & 9.1 & 1.0 & 2.00 & 0.23 \\
\hline
\end{tabular}




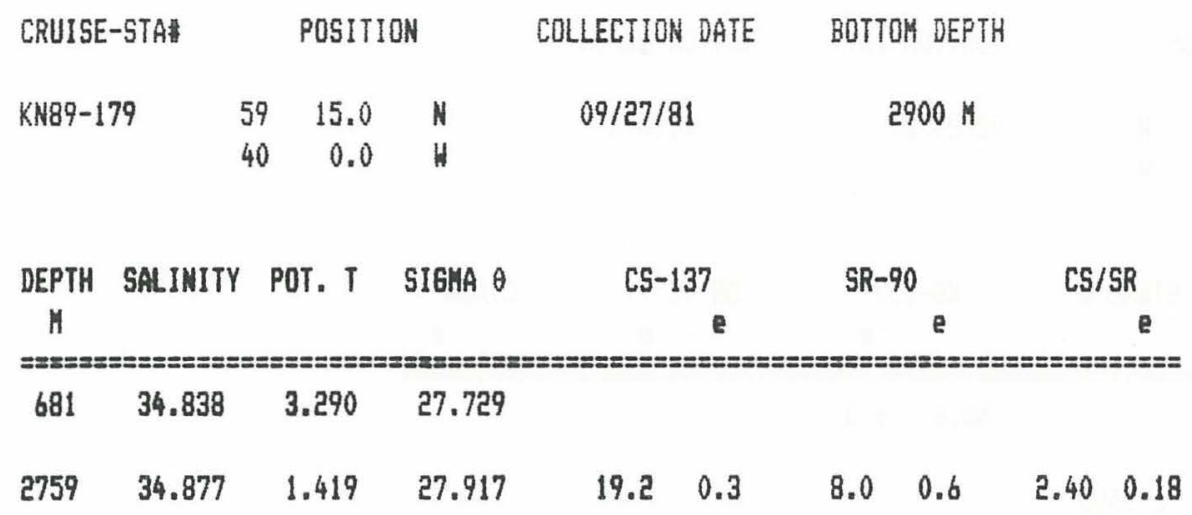

$\begin{array}{llcccc}\text { CRUISE-STA\# } & & \text { POSITION } & & \text { COLLECTION DATE } & \text { BOTTOH DEPTH } \\ \text { KN89-181 } & 59 & 39.0 & \text { N } & 08 / 27 / 81 & 2444\end{array}$

\begin{tabular}{|c|c|c|c|c|c|c|c|c|c|}
\hline DEPTH & SALINITY & POT. T & SIGMA $\theta$ & CS- & & SR- & & CS/ & \\
\hline Y & & & & & e & & e & & e \\
\hline$= \pm= \pm=0$ & 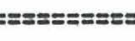 & 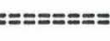 & $=2 x= \pm=x= \pm=$ & $== \pm= \pm=$ & $== \pm=0$ & $==x= \pm=$ & $= \pm= \pm=$ & $== \pm= \pm=x$ & $==x==$ \\
\hline 0 & & & & 26.2 & 6.9 & & & & \\
\hline 1 & & & & & & & & & \\
\hline 255 & 34.952 & 4.470 & 27.698 & & & & & & \\
\hline 752 & 34.874 & 3.550 & 27.732 & 15.2 & 0.3 & 8.4 & 0.3 & 1.81 & 0.07 \\
\hline 1443 & 34.922 & 3.404 & 27.784 & 10.9 & 0.2 & 6.9 & 0.5 & 1.58 & 0.12 \\
\hline 1937 & 34.925 & 2.698 & 27.852 & 12.0 & 0.2 & 8.2 & 0.2 & 1.46 & 0.04 \\
\hline 2300 & 34.882 & 1.731 & 27.898 & 18.0 & 0.1 & & & & \\
\hline 2379 & 34.879 & 1.490 & 27.914 & 18.7 & 0.3 & 10.1 & 0.1 & 1.85 & 0.03 \\
\hline 2418 & 34.880 & 1.448 & 27.917 & 18.7 & 0.3 & 9.9 & 0.5 & 1.89 & 0.10 \\
\hline
\end{tabular}

Q:1 SIGHA COUNTING UNCERTAINTY

CONCENTRATIONS ARE DPH/100 KG AS OF COLLECTION DATE 


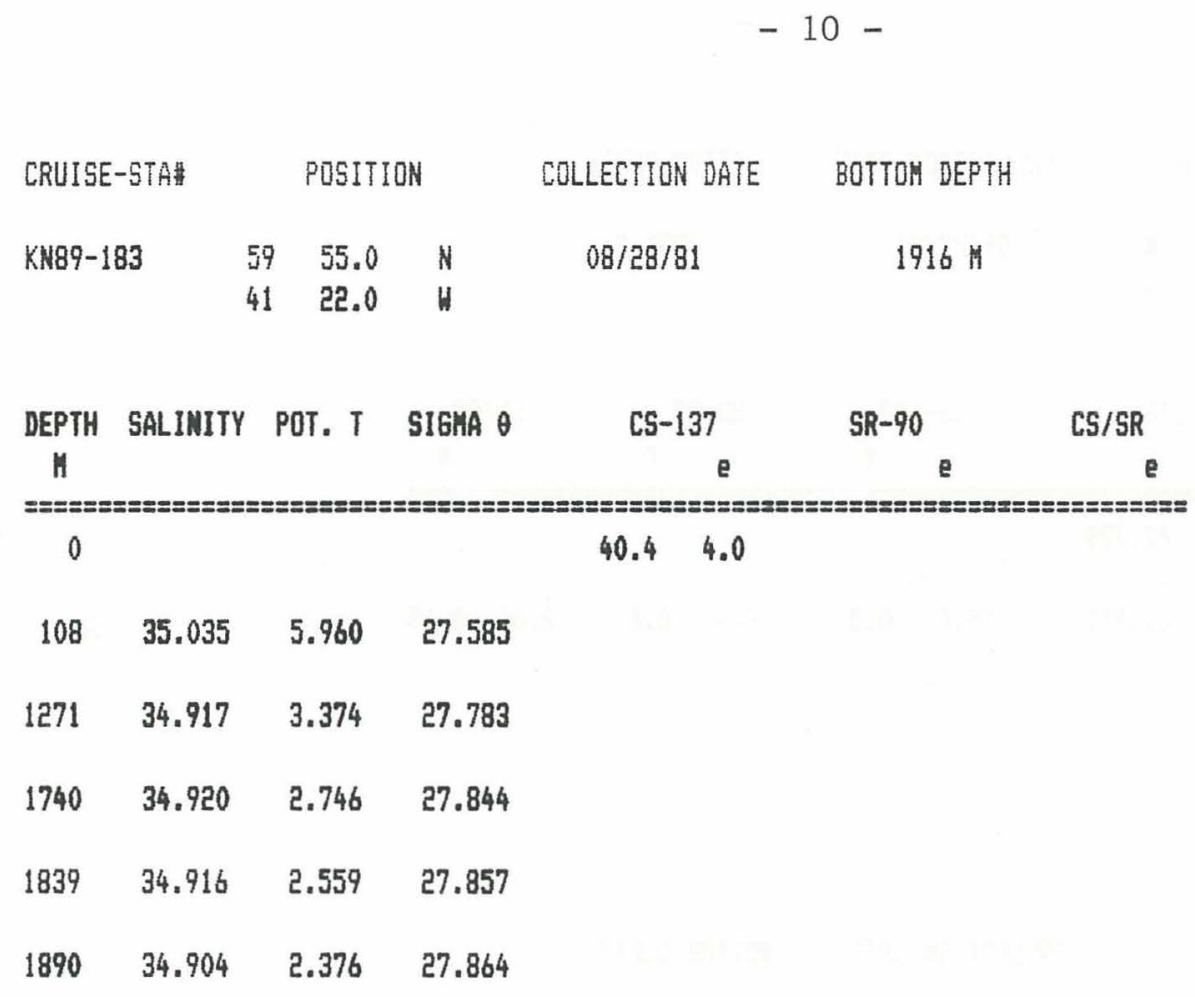

$\begin{array}{lrrrcc}\text { CRUISE-STA } & & \text { POSITION } & \text { COLLECTION DATE } & \text { BOTTOH DEPTH } \\ \text { KNB9-186 } & 60 & 5.0 & \text { N } & 08 / 29 / 81 & 242 ~ \\ & 42 & 11.0 & \text { H } & & \end{array}$

$\begin{array}{ccccc}\text { DEPTH SALINITY POT. T SIGHA } \theta & \text { CS-137 } & \text { SR-90 } & \text { CS/SR } \\ \text { H } & & \text { e } & \text { e } & \end{array}$

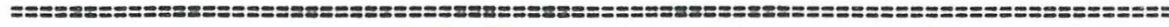

$29 \quad 33.446 \quad 1.410 \quad 26.769$

$\begin{array}{llllllllll}150 & 34.457 & 2.860 & 27.464 & 23.8 & 0.3 & 13.1 & 0.1 & 1.82 & 0.03\end{array}$

E:1 SIGHA COUNTING UNCERTAINTY

CONCENTRATIONS ARE DPM/100 KG AS OF COLLECTION DATE 


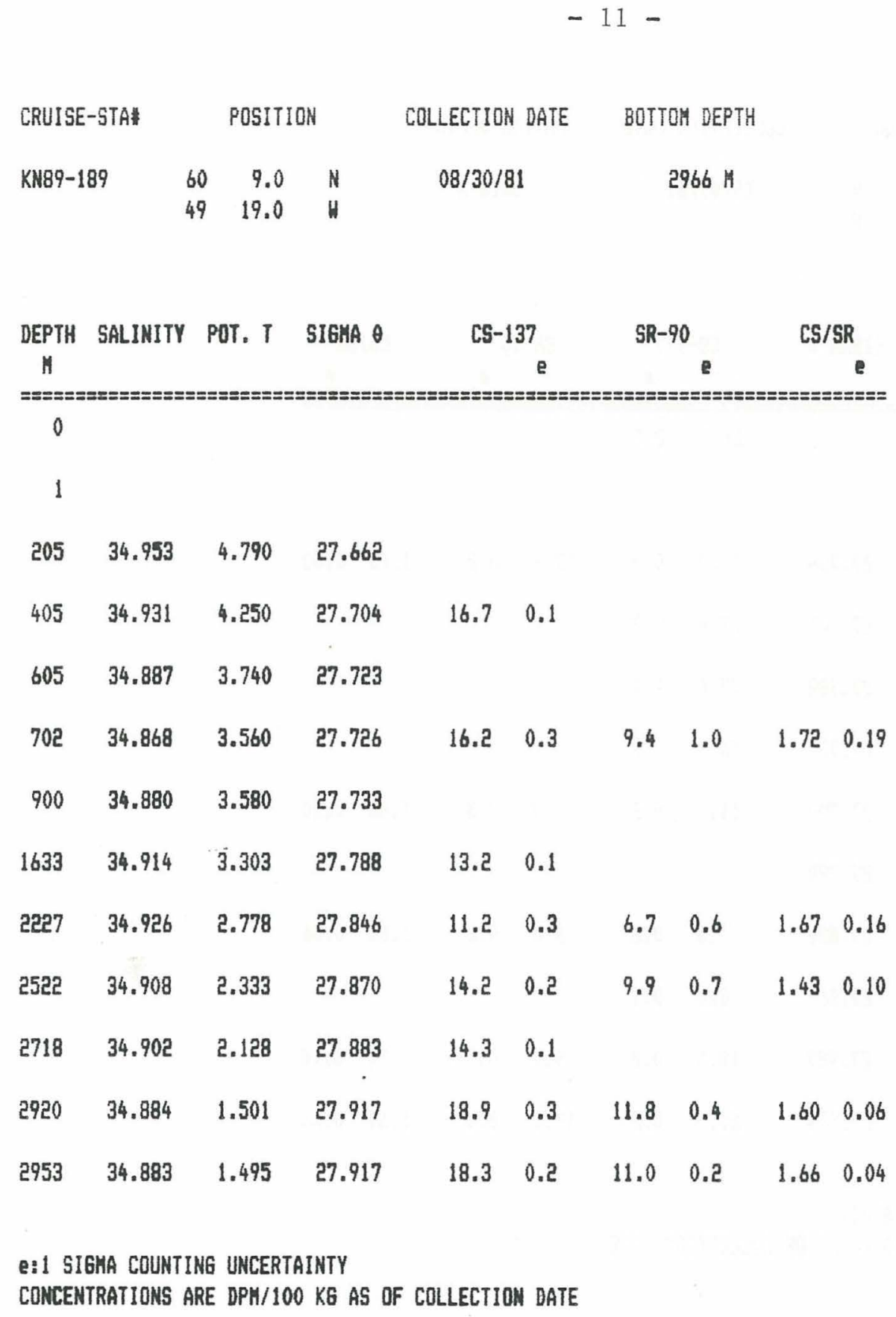




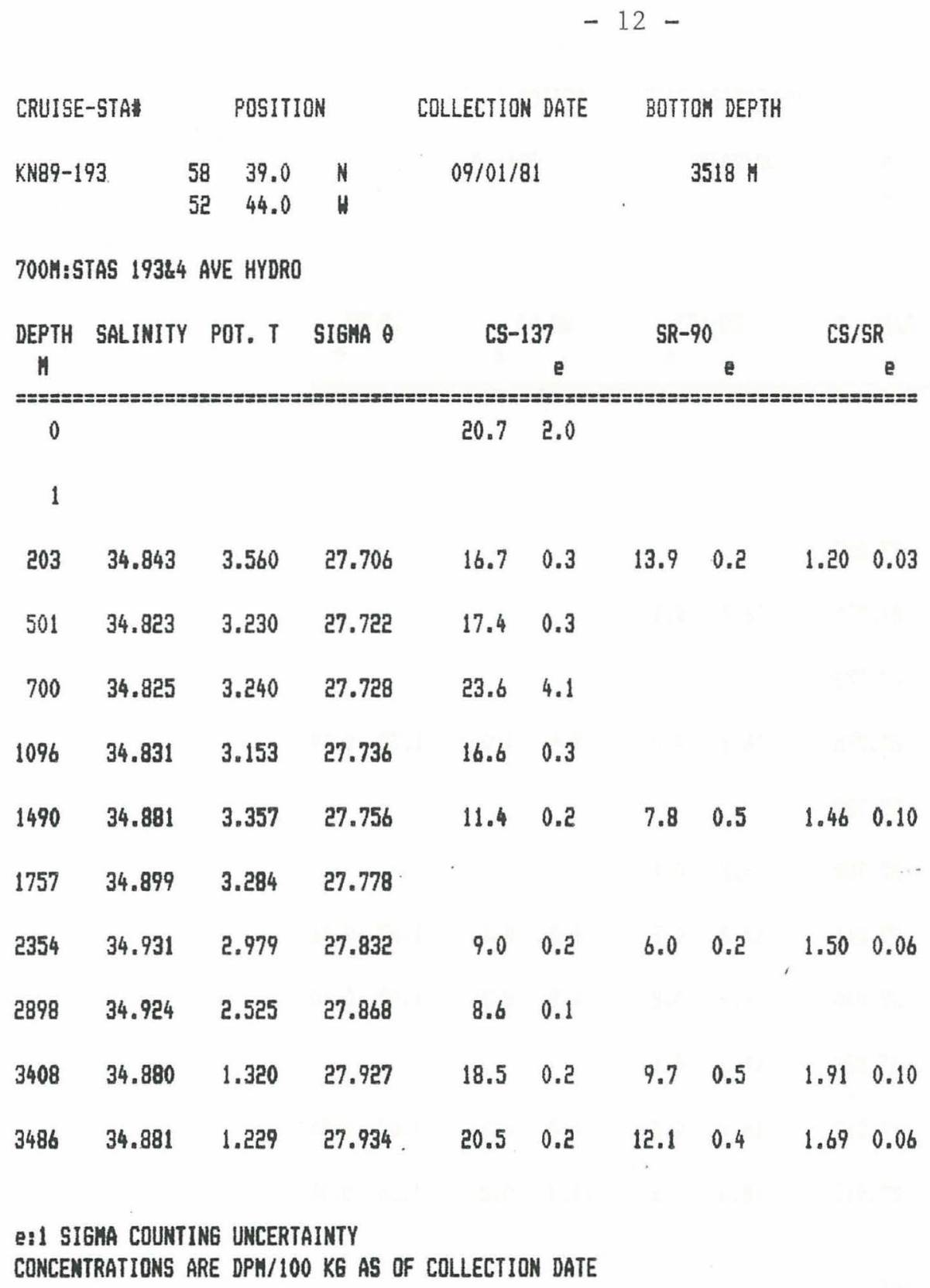




\begin{tabular}{llcccc} 
CRUISE-STA & \multicolumn{3}{c}{ POSITION } & COLLECTION DATE & BOTTOH DEPTH \\
KN89-197 & 56 & 54.0 & N & $09 / 03 / 81$ & $1986 \mathrm{H}$ \\
& 57 & 34.0 & H & &
\end{tabular}

SURF FRON STA 196

$\begin{array}{cccccc}\text { DEPTH SALINITY POT. T } & \text { SIGMA } \theta & \text { CS-137 } & \text { SR-90 } & \text { CS/SR }\end{array}$

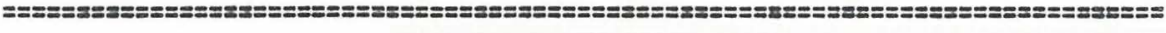

0

$202 \quad 34.812 \quad 3.620 \quad 27.675$

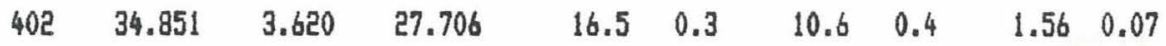

$\begin{array}{llllllllll}605 & 34.860 & 3.570 & 27.718 & 15.7 & 0.2 & 14.6 & 0.2 & 1.08 & 0.02\end{array}$

$\begin{array}{llllllllll}1003 & 34.870 & 3.421 & 27.741 & 15.9 & 0.2 & 10.8 & 0.1 & 1.47 & 0.02\end{array}$

$\begin{array}{llllllllll}1300 & 34.895 & 3.379 & 27.765 & 12.6 & 0.3 & 7.5 & 0.2 & 1.68 & 0.06\end{array}$

$\begin{array}{llllllllll}1700 & 34.915 & 3.155 & 27.803 & 13.1 & 0.3 & 7.5 & 0.5 & 1.75 & 0.12\end{array}$

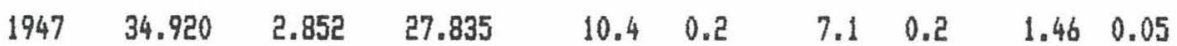

E: 1 SIGMA COUNTING UNCERTAINTY

CONCENTRATIONS ARE DPM/100 KG AS OF COLLECTION DATE 


$$
-14-
$$

$\begin{array}{llcccc}\text { CRUISE-STA\# } & & \text { POSITION } & \text { COLLECTION DATE } & \text { BOTTOM DEPTH } \\ \text { KN89-199 } & 56 & 36.0 & \text { N } & 09 / 03 / 81 & 434 \text { H } \\ & 59 & 20.0 & \text { H } & & \end{array}$

SURF FROM STAS 198-200

$\begin{array}{lllll}\text { DEPTH SALINITY POT. T SIGHA } \theta & \text { CS-137 } & \text { SR-90 } & \text { CS/SR } \\ \text { II } & & & & \end{array}$

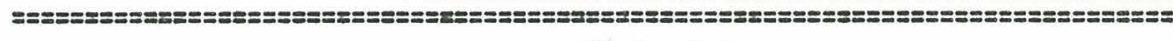

$\begin{array}{rrrr}66 & 32.946 & 0.180 & 26.439 \\ 153 & 33.664 & 0.450 & 27.004 \\ 353 & 34.751 & 3.660 & 27.622\end{array}$

Q:1 SIGMA COUNTING UNCERTAINTY CONCENTRATIONS ARE DPH/100 KG AS OF COLLECTION DATE 


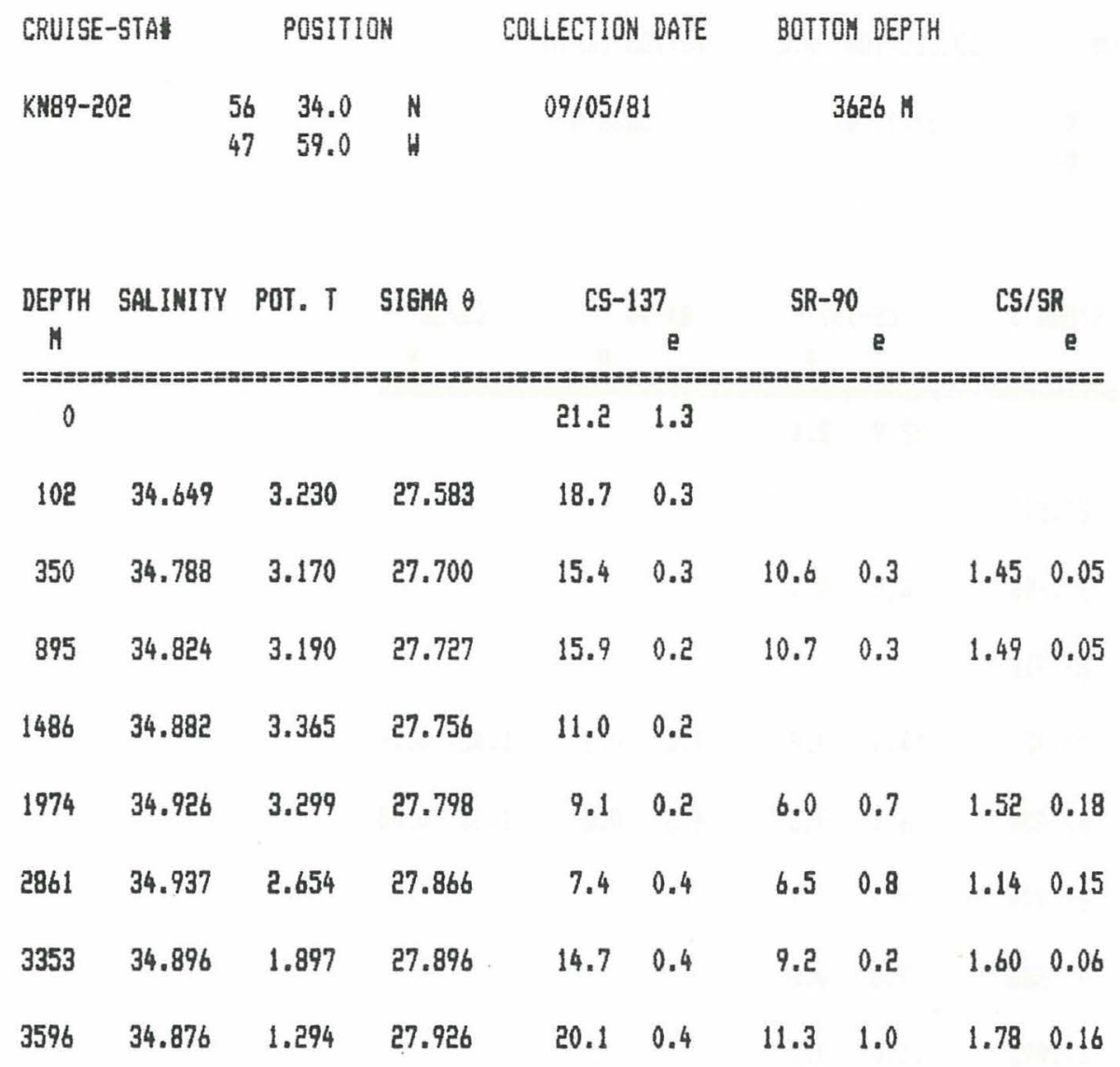

E:1 SIGMA COUNTING UNCERTAINTY

CONCENTRATIONS ARE DPH/100 KG AS OF COLLECTION DATE 


\begin{tabular}{llllcr} 
CRUISE-STAF & \multicolumn{3}{c}{ POSITION } & COLLECTION DATE & BOTTOH DEPTH \\
KN89-205 & 54 & 35.0 & $\mathrm{~N}$ & $09 / 07 / 81$ & $3636 \mathrm{H}$ \\
& 41 & 54.0 & $\mathrm{H}$ & &
\end{tabular}

2765M AVE OF 6 HYDRO

\begin{tabular}{|c|c|c|c|c|c|c|c|c|c|}
\hline $\begin{array}{l}\text { DEPTH } \\
====\end{array}$ & SALIMITY & POT. T & SIGMA $\theta$ & \multicolumn{2}{|c|}{ CS-137 } & \multicolumn{2}{|c|}{ SR-90 } & $\begin{array}{c}\text { CS/ } \\
== \pm=8\end{array}$ & $\begin{array}{c}\text { /SR } \\
\text { e } \\
\text { sez=: }\end{array}$ \\
\hline 0 & & & & 22.9 & 2.6 & & & & \\
\hline 151 & 34.749 & 4.080 & 27.578 & & & & & & \\
\hline 601 & 34.823 & 3.580 & 27.688 & 16.0 & 0.1 & & & & \\
\hline 749 & 34.829 & 3.390 & 27.712 & & & & & & \\
\hline 994 & 34.840 & 3.333 & 27.726 & 14.6 & 0.2 & 9.0 & 0.2 & 1.62 & 0.04 \\
\hline 2467 & 34.952 & 2.917 & 27.854 & 6.1 & 0.3 & 4.5 & 0.8 & 1.36 & 0.25 \\
\hline 2765 & 34.958 & 2.750 & 27.874 & 9.3 & 1.6 & & & & \\
\hline 2956 & 34.960 & 2.638 & 27.886 & 7.3 & 0.2 & & & & \\
\hline 3443 & 34.934 & 2.286 & 27.895 & 11.0 & 0.1 & & & & \\
\hline 3538 & 34.927 & 2.183 & 27.898 & 9.3 & 0.3 & 6.9 & 0.8 & 1.35 & 0.16 \\
\hline
\end{tabular}

E:1 SIGMA COUNTING UNCERTAINTY

CONCENTRATIONS ARE DPM/100 KG AS OF COLLECTION DATE 


$\begin{array}{llllcr}\text { CRUISE-STA } & & \text { POSITION } & \text { COLLECTION DATE } & \text { BOTTOH DEPTH } \\ \text { KN89-211 } & 47 & 39.0 & \text { N } & 09 / 11 / 81 & 4559 \mathrm{M} \\ & 37 & 50.0 & \mathrm{H} & & \end{array}$

\begin{tabular}{|c|c|c|c|c|c|c|c|}
\hline $\begin{array}{l}\text { DEPTH } \\
\text { H }\end{array}$ & SALINITY & POT. T & SIGHA $\theta$ & CS- & 37 & SR-90 & $\begin{array}{r}\text { CS/SR } \\
\mathrm{e}\end{array}$ \\
\hline$s=x=x:$ & $8=1=x=8=$ & $z=x=x=4$ & $y=x= \pm=x$ & $== \pm==x$ & $== \pm=$ & $== \pm==x=$ & m=x=x=x=x \\
\hline 0 & & & & 24.5 & 1.1 & & \\
\hline 1190 & 34.926 & 4.116 & 27.715 & & & & \\
\hline 2400 & & & & 9.9 & 1.0 & & \\
\hline 3274 & 34.938 & 2.633 & 27.869 & & & & \\
\hline 4399 & 34.902 & 1.841 & 27.906 & & & & \\
\hline 4497 & 34.909 & 1.842 & 27.911 & & & & \\
\hline
\end{tabular}

E:1 SIGMA COUNTING UNCERTAINTY

CONCENTRATIONS ARE DPH/100 KG AS OF COLLECTION DATE 


$\begin{array}{lrrrcr}\text { CRUISE-STAE } & & \text { POSITION } & \text { COLLECTION DATE } & \text { BOTTOH DEPTH } \\ \text { KNB9-214 } & 50 & 59.0 & N & 09 / 14 / 81 & 4278 \text { H } \\ & 43 & 0.0 & \text { N } & & \end{array}$

\begin{tabular}{|c|c|c|c|c|c|c|c|c|c|}
\hline $\begin{array}{c}\text { DEPTH } \\
\text { M }\end{array}$ & SALINITY & POT. T & SIGMA $\theta$ & & CS-137 & \multicolumn{2}{|c|}{ SR-90 } & \multicolumn{2}{|c|}{ CS/SR } \\
\hline$=3 x=2=$ & 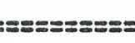 & $==E=E=x= \pm$ & $z=x==\pi=x=$ & $==2=2=$ & $==x=-$ & $== \pm==x$ & $== \pm==$ & $y==x=$ & $s=x==$ \\
\hline 7 & & & & 22.4 & 0.2 & & & & \\
\hline 201 & 35.754 & 13.180 & 26.942 & 20.7 & 0.4 & 11.2 & 0.5 & 1.85 & 0.09 \\
\hline 397 & 35.673 & 12.580 & 26.998 & 19.7 & 0.1 & & & & \\
\hline 591 & 35.078 & 8.230 & 27.300 & 10.1 & 0.3 & 6.4 & 0.3 & 1.58 & 0.09 \\
\hline 975 & 34.888 & 4.080 & 27.688 & 12.4 & 0.3 & 7.6 & 0.8 & 1.63 & 0.18 \\
\hline 1356 & 34.919 & 3.814 & 27.741 & 8.7 & 0.1 & & & & \\
\hline 1540 & 34.913 & 3.623 & 27.755 & 11.5 & 0.2 & 8.1 & 0.5 & 1.42 & 0.09 \\
\hline 2349 & 34.939 & 3.195 & 27.818 & 10.8 & 0.1 & & & & \\
\hline 2672 & 34.934 & 2.906 & 27.841 & 7.8 & 0.3 & & & & \\
\hline 3163 & 34.931 & 2.441 & 27.880 & 6.7 & 0.4 & 3.8 & 0.5 & 1.76 & 0.25 \\
\hline 3753 & 34.904 & 1.911 & 27.902 & 9.4 & 0.2 & 6.0 & 0.7 & 1.57 & 0.19 \\
\hline 4098 & 34.892 & 1.664 & 27.911 & 14.9 & 0.2 & & & & \\
\hline 4198 & 34.881 & 1.522 & 27.913 & 15.2 & 0.3 & 8.6 & 0.6 & 1.77 & 0.13 \\
\hline 4244 & 34.880 & 1.470 & 27.916 & 15.4 & 0.3 & 8.7 & 0.5 & 1.77 & 0.11 \\
\hline
\end{tabular}

E:1 SIGMA COUNTING UNCERTAINTY

CONCENTRATIONS ARE DPH/100 KG AS OF COLLECTION DATE 


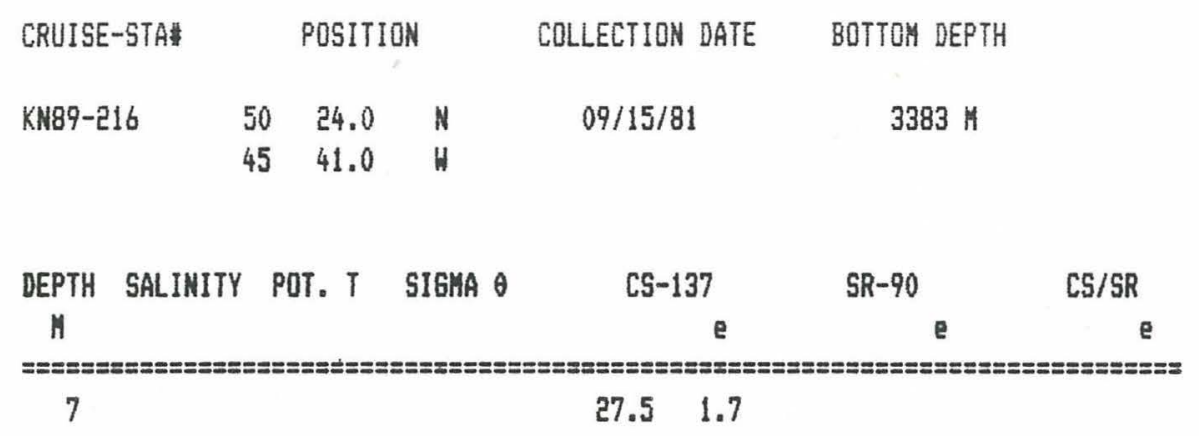

$\begin{array}{llllcr}\text { CRUISE-STA } & & \text { POSITION } & \text { COLLECTION DATE } & \text { BOTTOH DEPTH } \\ \text { KN89-218 } & 49 & 45.0 & \text { N } & 09 / 16 / 81 & 2132 \text { M } \\ & 48 & 35.0 & \text { H } & & \end{array}$

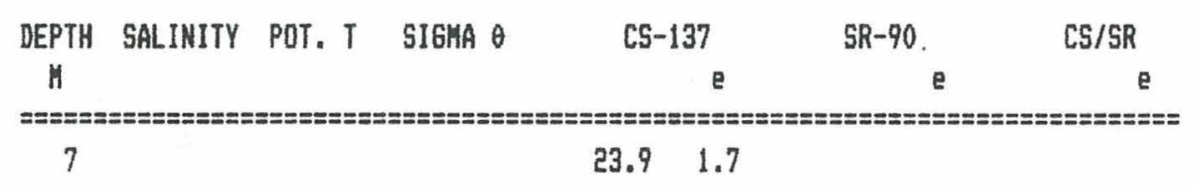

P:1 SIGMA COUNTING UNCERTAINTY

CONCENTRATIONS ARE DPH/100 KG AS OF COLLECTION DATE 
$-20-$ 
R/V HUDSON WINTER CRUISE TO THE GREENLAND SEA, 1982

This cruise, in late winter 1982, was designed to sample the Greenland sea in winter conditions to attempt to observe deep convective mixing in action. 


\begin{tabular}{|c|c|c|c|c|c|c|c|c|c|}
\hline \multicolumn{2}{|c|}{ CRUISE-STAL } & \multicolumn{2}{|c|}{ POSITION } & \multicolumn{2}{|c|}{ COLLECTION DATE } & \multicolumn{2}{|c|}{ BOTTOM DEPTH } & & \\
\hline \multicolumn{2}{|c|}{ HUD82-71 } & 32.1 & N & \multicolumn{2}{|c|}{$03 / 20 / 82$} & \multicolumn{2}{|c|}{$1494 \mathrm{M}$} & & \\
\hline DEPTH & SALINITY & POT. T & SIGHA $\theta$ & \multicolumn{2}{|c|}{ C5-137 } & \multicolumn{2}{|c|}{ SR-90 } & \multicolumn{2}{|c|}{ CS/SR } \\
\hline H & & & & & e & & e & & e \\
\hline \multicolumn{10}{|c|}{ 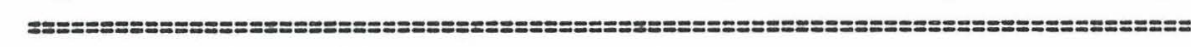 } \\
\hline 7 & 35.078 & 4.665 & 27.776 & 69.7 & 0.3 & & & & \\
\hline 47 & 35.077 & 4.653 & 27.777 & 85.3 & 0.8 & 25.0 & 0.7 & 3.41 & 0.10 \\
\hline 116 & 35.085 & 4.186 & 27.835 & 60.5 & 0.6 & & & & \\
\hline 185 & 35.063 & 3.659 & 27.871 & 61.0 & 0.7 & 19.5 & 0.6 & 3.13 & 0.10 \\
\hline 275 & 35.066 & 3.404 & 27.899 & 69.9 & 0.6 & & & & \\
\hline 385 & 35.050 & 2.931 & 27.931 & 61.3 & 0.7 & 25.3 & 0.5 & 2.42 & 0.06 \\
\hline 547 & 34.939 & 1.380 & 27.970 & 37.7 & 0.6 & & & & \\
\hline 742 & 34.912 & -0.390 & 28.055 & 11.2 & 0.4 & 5.7 & 0.4 & 1.96 & 0.15 \\
\hline 919 & 34.909 & -0.703 & 28.067 & 7.0 & 0.3 & & & & \\
\hline 1118 & 34.912 & -0.904 & 28.078 & 6.1 & 0.3 & 3.5 & 0.4 & 1.74 & 0.22 \\
\hline 1266 & 34.909 & -0.985 & 28.079 & 3.2 & 0.2 & & & & \\
\hline 1416 & 34.910 & -1.029 & 28.082 & 6.0 & 0.6 & 3.3 & 0.4 & 1.82 & 0.29 \\
\hline 1486 & 34.909 & -1.063 & 28.082 & 3.2 & 0.3 & 3.1 & 0.5 & 1.03 & 0.19 \\
\hline
\end{tabular}




\begin{tabular}{|c|c|c|c|c|c|c|c|c|c|}
\hline \multirow{2}{*}{\multicolumn{2}{|c|}{$\begin{array}{l}\text { CRUISE-STAN } \\
\text { HUD82-84 }\end{array}$}} & \multicolumn{2}{|c|}{ POSITION } & \multirow{2}{*}{\multicolumn{2}{|c|}{$\begin{array}{c}\text { COLLECTION DATE } \\
03 / 22 / 82\end{array}$}} & \multirow{2}{*}{\multicolumn{4}{|c|}{$\begin{array}{r}\text { BOTTOM DEPTH } \\
1790 \mathrm{H}\end{array}$}} \\
\hline & & $\begin{array}{ll}70 & 1.4\end{array}$ & N & & & & & & \\
\hline \multirow{2}{*}{$\begin{array}{c}\text { DEPTH } \\
n\end{array}$} & \multirow[t]{2}{*}{ SALINITY } & \multirow[t]{2}{*}{ POT. T } & \multirow[t]{2}{*}{ SIGHA $\theta$} & \multicolumn{2}{|c|}{ C5-137 } & \multicolumn{2}{|c|}{ SR-90 } & \multicolumn{2}{|c|}{ CS/SR } \\
\hline & & & & & e & & e & & e \\
\hline & $z==\varepsilon= \pm t$ & $=\boldsymbol{x = x = x}$ & $z=x==z=$ & $==x=-x=-x=$ & $z===x=$ & 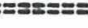 & $==\mathbf{z = = -}$ & 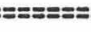 & :=:= \\
\hline 5 & 34.712 & 5.468 & 27.391 & 189.0 & 0.5 & 43.0 & 1.0 & 4.40 & 0.10 \\
\hline 130 & & & & 108.7 & 0.7 & 34.1 & 0.5 & 3.19 & 0.05 \\
\hline 337 & 35.145 & 5.821 & 27.690 & 59.8 & 0.5 & 22.0 & 0.3 & 2.72 & 0.04 \\
\hline 692 & 34.938 & 1.411 & 27.967 & 30.2 & 0.5 & 11.3 & 0.5 & 2.67 & 0.13 \\
\hline 1073 & 34.912 & -0.697 & 28.069 & 9.2 & 0.4 & & & & \\
\hline 1265 & 34.911 & -0.870 & 28.076 & 6.6 & 0.8 & 2.9 & 0.3 & 2.28 & 0.36 \\
\hline 1413 & 34.910 & -0.922 & 28.077 & 5.2 & 0.6 & 4.0 & 0.3 & 1.30 & 0.18 \\
\hline 1616 & 34.911 & -0.984 & 28.081 & 6.1 & 0.7 & 3.8 & 0.4 & 1.61 & 0.25 \\
\hline 1716 & 34.909 & -1.030 & 28.081 & 6.0 & 0.6 & & & & \\
\hline
\end{tabular}


- $24-$ 
ARKTIS II-3: R/V POLARSTERN 1984 CRUISE IN NORTHERN FRAM STRAIT

This cruise provided an opportunity to sample Atlantic water circulating in the Nansen Basin, Eurasian Basin Deep Water, and deep water flowing into the northern Greenland sea from the Arctic Basin. 


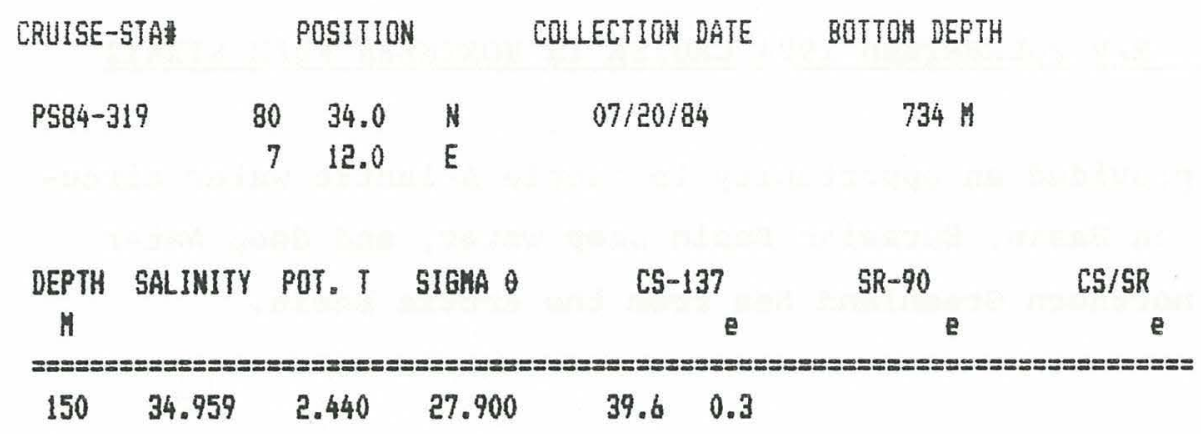

$\begin{array}{llcccc}\text { CRUISE-STA } & & \text { POSITION } & \text { COLLECTION DATE } & \text { BOTTOM DEPTH } \\ \text { PS84-325 } & 81 & 18.0 & N & 07 / 22 / 84 & \text { 2272 H } \\ & 15 & 23.0 & E & & \end{array}$

\begin{tabular}{|c|c|c|c|c|c|c|c|c|c|}
\hline \multirow{2}{*}{$\begin{array}{c}\text { DEPTH } \\
\text { H }\end{array}$} & \multirow[t]{2}{*}{ SALINITY } & \multirow[t]{2}{*}{ POT. $T$} & \multirow[t]{2}{*}{ SIGKA $\theta$} & \multicolumn{2}{|c|}{ CS- 137} & \multicolumn{2}{|c|}{$5 R-90$} & \multicolumn{2}{|c|}{ CS/SR } \\
\hline & & & & & e & & e & & e \\
\hline$= \pm=x=x$ & 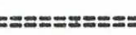 & 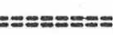 & $y=2 x=x=8=$ & $y=x=x=$ & $=e=x=$ & $== \pm= \pm x$ & $=x=x$ & $E== \pm=x$ & $== \pm=x$ \\
\hline 5 & 32.952 & & & 59.3 & 0.9 & 15.8 & 0.9 & 3.75 & 0.22 \\
\hline 67 & 34.637 & 1.006 & 27.753 & 52.0 & 0.7 & 15.3 & 0.4 & 3.40 & 0.10 \\
\hline 143 & 34.901 & 2.412 & 27.858 & 55.9 & 1.5 & 16.2 & 0.3 & 3.45 & 0.11 \\
\hline 190 & 34.872 & 1.888 & 27.878 & 57.6 & 0.8 & 16.3 & 1.6 & 3.53 & 0.35 \\
\hline 362 & 35.000 & 2.567 & 27.924 & 45.0 & 0.9 & & & & \\
\hline 590 & 34.959 & 1.398 & 27.985 & 38.8 & 0.7 & & & & \\
\hline 787 & 34.926 & 0.314 & 28.029 & 20.6 & 0.4 & 9.4 & 0.3 & 2.19 & 0.08 \\
\hline 987 & 34.920 & -0.320 & 28.058 & 12.2 & 0.3 & 6.2 & 0.3 & 1.97 & 0.11 \\
\hline 1300 & 34.922 & & & 7.4 & 0.2 & & & & \\
\hline$\$ 600$ & 34.922 & & & 7.8 & 0.1 & & & & \\
\hline 1601 & & & & 8.1 & 0.2 & 4.4 & 0.1 & 1.84 & 0.06 \\
\hline 2090 & 34.929 & & & 3.0 & 0.2 & 1.2 & 0.2 & 2.50 & 0.45 \\
\hline
\end{tabular}




\begin{tabular}{|c|c|c|c|c|c|}
\hline \multirow{2}{*}{$\begin{array}{l}\text { CRUISE-STA\# } \\
\text { PS84-327 }\end{array}$} & \multicolumn{2}{|c|}{ POSITION } & COLLECTION DATE & BOTTOH DEPTH & \\
\hline & $\begin{array}{r}8.5 \\
43.7\end{array}$ & $\begin{array}{l}N \\
E\end{array}$ & $07 / 23 / 84$ & $1028 M$ & \\
\hline DEPTH SALINITY & POT. T & SIGHA $\theta$ & es-137 & e & $\begin{array}{c}\text { CS/SR } \\
\text { e }\end{array}$ \\
\hline $5 \quad 34.849$ & & & 69.7 & 19.9 & $\begin{array}{ll}3.50 & 0.07\end{array}$ \\
\hline
\end{tabular}

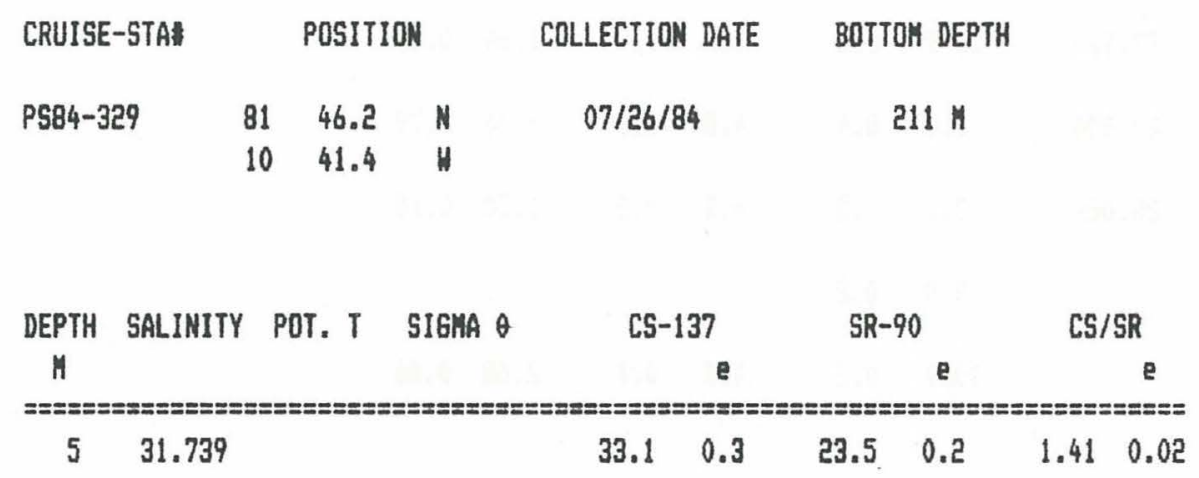

Q:1 SIGMA COUNTING UNCERTAINTY

CONCENTRATIONS ARE DPM/100 KG AS OF COLLECTION DATE 


\begin{tabular}{|c|c|c|c|c|c|c|c|c|c|}
\hline \multicolumn{2}{|c|}{ CRUISE-STA\# } & \multicolumn{2}{|c|}{ POSITION } & \multicolumn{2}{|c|}{ COLLECTION JATE } & \multicolumn{2}{|c|}{ ВОTTOH DEPTH } & & \\
\hline \multicolumn{2}{|c|}{ P584-331 } & 56.0 & N & \multicolumn{2}{|c|}{$07 / 26 / 94$} & \multicolumn{3}{|c|}{$2450 \mathrm{H}$} & \\
\hline DEPTH & SALINITY & POT. T & SIGMA $\theta$ & $\operatorname{cs}-1$ & & $S R-$ & & CS/ & $S R$ \\
\hline$n$ & & & & & e & & e & & e \\
\hline \multicolumn{10}{|c|}{ 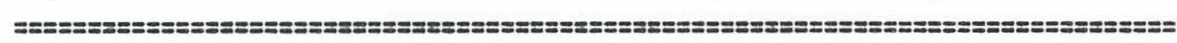 } \\
\hline 5 & 31.637 & & & 31.2 & 0.3 & 23.2 & 0.2 & 1.34 & 0.02 \\
\hline 161 & 34.510 & -0.349 & 27.728 & 19.6 & 0.4 & 11.7 & 0.3 & 1.68 & 0.06 \\
\hline 202 & 34.744 & 0.308 & 27.883 & 20.5 & 0.3 & 12.0 & 0.7 & 1.71 & 0.10 \\
\hline 230 & 34.800 & 0.387 & 27.923 & 13.0 & 0.4 & 8.1 & 0.5 & 1.60 & 0.11 \\
\hline 300 & 34.860 & 0.571 & 27.960 & 15.1 & 0.4 & 9.2 & 0.4 & 1.64 & 0.08 \\
\hline 498 & 34.884 & 0.329 & 27.994 & 7.0 & 0.4 & 4.8 & 0.9 & 1.46 & 0.29 \\
\hline 796 & 34.896 & 0.034 & 28.020 & 7.1 & 0.3 & 4.5 & 0.2 & 1.59 & 0.10 \\
\hline 1150 & 34.909 & & & 9.0 & 0.2 & & & & \\
\hline 1525 & 34.925 & & & 10.0 & 0.2 & 4.8 & 0.1 & 2.08 & 0.06 \\
\hline 2150 & 34.935 & & & & & & & & \\
\hline 2350 & 34.931 & & & 7.0 & 0.2 & 2.8 & 0.2 & 2.50 & 0.19 \\
\hline
\end{tabular}

$\begin{array}{lrrrcc}\text { CRUISE-STA } & & \text { POSITION } & \text { COLLECTION DATE } & \text { BOTTOM DEPTH } \\ \text { PS84-332 } & 82 & 4.7 & \text { N } & 07 / 26 / 84 & 2806 \mathrm{n}\end{array}$

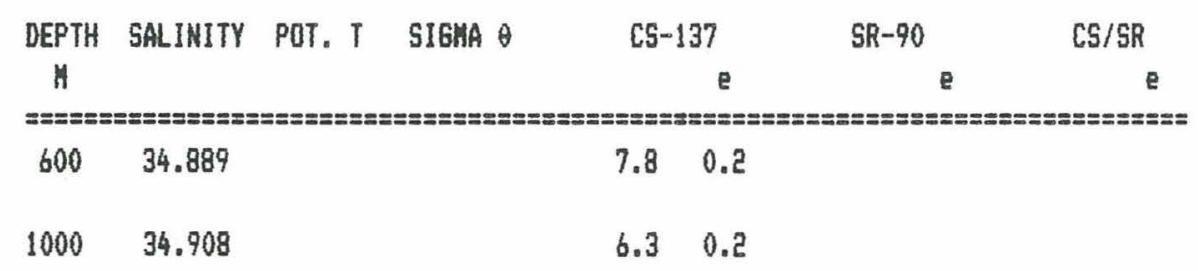

Q:1 SIGHA COUNTING UNCERTAINTY

CONCENTRATIONS ARE DPM/100 KG AS OF COLLECTION DATE 


\begin{tabular}{|c|c|c|c|c|c|c|c|c|c|}
\hline \multicolumn{2}{|c|}{ CRUISE-STA\# } & \multicolumn{2}{|c|}{ POSITION } & \multicolumn{2}{|c|}{ CQLLECTION DATE } & \multicolumn{4}{|c|}{ ВОTTOM DEPTH } \\
\hline \multicolumn{2}{|c|}{ PS84-334 } & 32.0 & N & \multicolumn{2}{|c|}{$07 / 27 / 84$} & \multicolumn{4}{|c|}{$4343 \mathrm{H}$} \\
\hline $\begin{array}{c}\text { DEPTH } \\
M\end{array}$ & SALINITY & POT. T & SIGMA $\theta$ & \multicolumn{2}{|c|}{$[5-137$} & \multicolumn{2}{|c|}{ SR-90 } & \multicolumn{2}{|c|}{ CS/SR } \\
\hline$== \pm$ & $y=z= \pm=z$ & $==x=1= \pm=1=$ & $== \pm=$ & $== \pm= \pm=z=8$ & $==2= \pm=$ & $== \pm=x=$ & $=:= \pm=x=$ & $== \pm=x$ & $== \pm=2$ \\
\hline 5 & 32.179 & & & 43.2 & 0.3 & 25.4 & 0.5 & 1.70 & 0.04 \\
\hline 236 & 34.885 & 1.579 & 27.912 & 46.6 & 0.4 & 15.9 & 0.4 & 2.93 & 0.08 \\
\hline 266 & 34.852 & 0.989 & 27.927 & 32.0 & 0.4 & 15.4 & 0.5 & 2.08 & 0.07 \\
\hline 304 & 34.931 & 1.540 & 27.952 & 38.3 & 0.5 & 14.8 & 0.4 & 2.59 & 0.08 \\
\hline 393 & 34.915 & 1.046 & 27.974 & 29.5 & 0.4 & 10.2 & 1.2 & 2.89 & 0.34 \\
\hline 504 & 34.923 & 0.852 & 27.993 & 26.5 & 0.4 & 9.7 & 1.5 & 2.73 & 0.42 \\
\hline 991 & 34.918 & -0.319 & 28.056 & 13.8 & 0.3 & 8.1 & 0.5 & 1.70 & 0.11 \\
\hline 1400 & 34.921 & & & 6.8 & 0.1 & & & & \\
\hline 1401 & & & & 6.2 & 0.2 & 3.9 & 0.2 & 1.59 & 0.10 \\
\hline 2400 & 34.926 & & & 3.6 & 0.1 & & & & \\
\hline 2401 & & & & 3.0 & 0.2 & 1.9 & 0.2 & 1.58 & 0.20 \\
\hline
\end{tabular}

CRUISE-STAI POSHTION COLLECTION DATE BOTTOM DEPTH

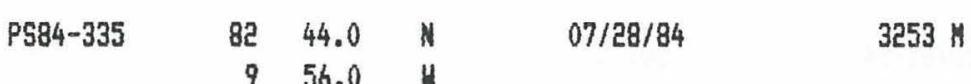

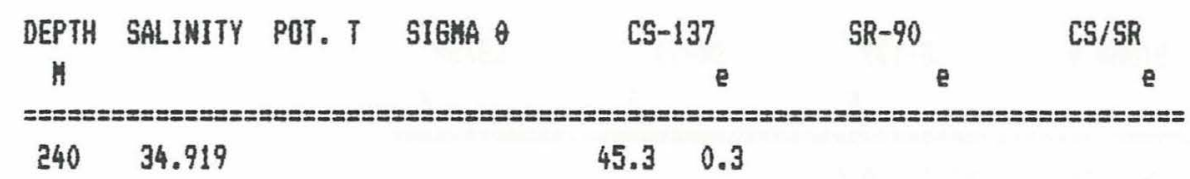

E:1 SIGHA COUNTING UNCERTAINTY

CONCENTRATIONS ARE DPM/100 KG AS OF COLLECTION DATE 


\begin{tabular}{|c|c|c|c|c|c|c|c|c|c|}
\hline \multicolumn{2}{|c|}{ CRUISE-STA\# } & \multicolumn{2}{|c|}{ POSITION } & \multicolumn{2}{|c|}{ COLLECTION DATE } & \multicolumn{4}{|c|}{ BOTTOM DEPTH } \\
\hline \multicolumn{2}{|c|}{$P 584-354$} & $\begin{array}{rr}31 & 29.0 \\
2 & 2.0\end{array}$ & $\begin{array}{l}N \\
H\end{array}$ & \multicolumn{2}{|c|}{$07 / 29 / 84$} & \multicolumn{4}{|c|}{$3154 M$} \\
\hline $\begin{array}{c}\text { DEPTH } \\
M\end{array}$ & SALINITY & POT. T & SIGMA $\theta$ & \multicolumn{2}{|c|}{ CS-137 } & SR- & 90 & \multicolumn{2}{|c|}{ CS/SR } \\
\hline \multicolumn{10}{|c|}{ 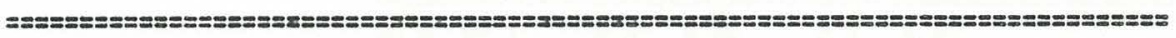 } \\
\hline 285 & 34.944 & 1.947 & 27.931 & 46.4 & 0.3 & 14.1 & 0.2 & 3.29 & 0.05 \\
\hline 370 & 34.974 & & & 36.6 & 0.3 & & & & \\
\hline
\end{tabular}

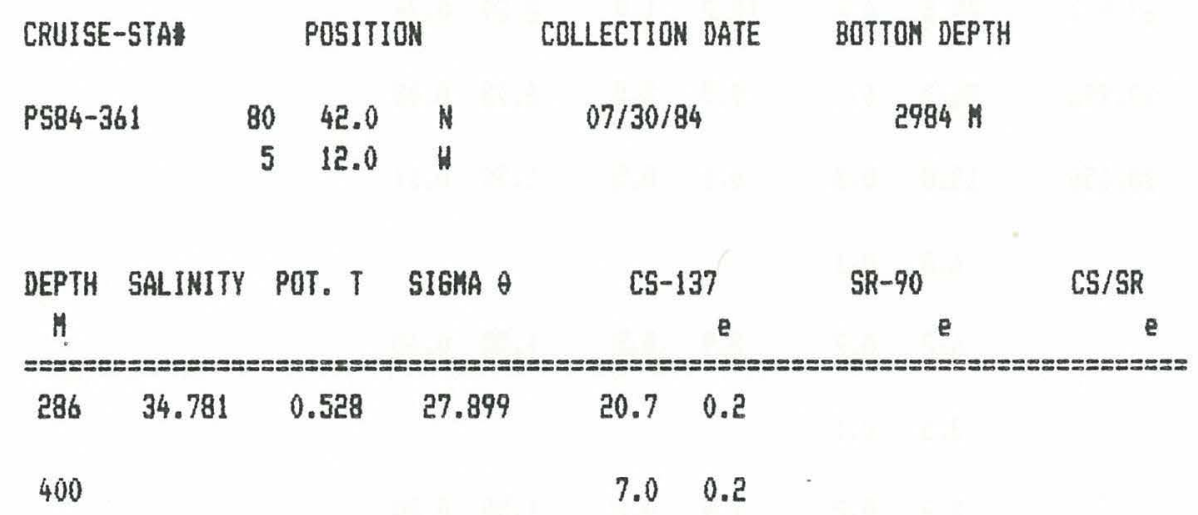

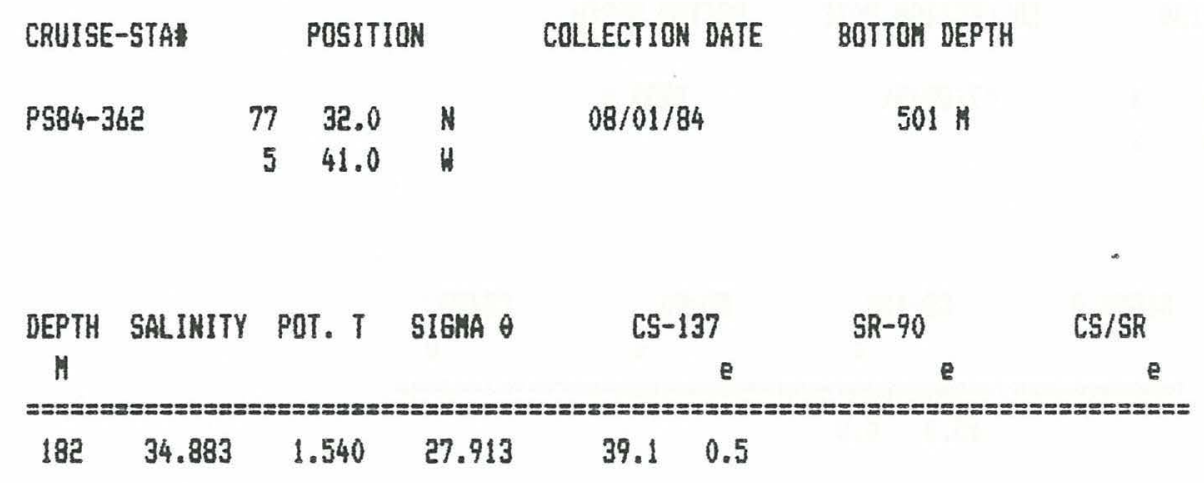




\begin{tabular}{|c|c|c|c|c|c|c|c|c|c|}
\hline \multicolumn{2}{|c|}{ CRUISE-STA } & \multicolumn{2}{|c|}{ POSITION } & \multicolumn{2}{|c|}{ COLLECTION DATE } & \multicolumn{4}{|c|}{ BOTTOH DEPTH } \\
\hline \multicolumn{2}{|c|}{ P584-363 } & $\begin{array}{rr}77 & 40.0 \\
4 & 56.0\end{array}$ & $\begin{array}{l}N \\
W\end{array}$ & \multicolumn{2}{|c|}{$08 / 01 / 84$} & \multicolumn{4}{|c|}{$1129 \mathrm{M}$} \\
\hline $\begin{array}{l}\text { DEPTH } \\
\text { H }\end{array}$ & SALINITY & POT. T & & CS-1 & & SR- & & CS/ & \\
\hline & & & & & & \multicolumn{4}{|c|}{ 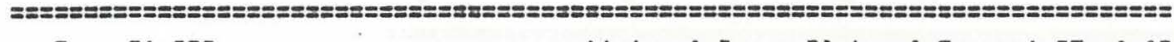 } \\
\hline 5 & 31.989 & & & 41.1 & 0.3 & 30.4 & 0.7 & 1.35 & 0.03 \\
\hline 177 & 34.892 & 1.698 & 27.909 & 37.4 & 0.6 & & & & \\
\hline 316 & 34.992 & 1.785 & 27.982 & 40.5 & 0.6 & & & & \\
\hline
\end{tabular}

\begin{tabular}{|c|c|c|c|c|c|}
\hline CRUISE-STAI & & OSIT! & & COLLECTION DATE & BOTTOM DEPTH \\
\hline P584-364 & 77 & 34.0 & $N$ & $08 / 02 / 84$ & 1539 月 \\
\hline & 4 & 43.0 & H & & \\
\hline
\end{tabular}

\begin{tabular}{|c|c|c|c|c|c|c|c|c|c|}
\hline $\begin{array}{c}\text { DEPTH } \\
\text { H }\end{array}$ & SALIMITY & РОT. T & SIGHA $\theta$ & CS- & 37 & SR- & e & CS/ & SR \\
\hline E=z=:= & 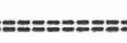 & E=:=:=تي & $=z= \pm=2=8$ & $== \pm=x$ & $z==z=$ & $== \pm==$ & $==x=z$ & $== \pm==$ & $==z=$ \\
\hline 5 & 32.176 & & & 42.6 & 0.4 & 27.2 & 0.3 & 1.57 & 0.02 \\
\hline 91 & 34.361 & -0.815 & 27.628 & 28.0 & 0.7 & & & & \\
\hline 215 & 34.973 & 2.241 & 27.930 & 44.4 & 0.4 & 14.6 & 0.8 & 3.04 & 0.17 \\
\hline 314 & 34.909 & 1.151 & 27.962 & 25.8 & 0.7 & & & & \\
\hline 342 & 35.013 & 1.982 & 27.983 & 42.3 & 1.5 & & & & \\
\hline 554 & 34.936 & 0.605 & 28.020 & 23.2 & 0.6 & & & & \\
\hline 772 & 34.905 & -0.271 & 28.043 & 28.1 & 0.5 & & & & \\
\hline 837 & 34.912 & -0.431 & 28.057 & 15.3 & 0.2 & & & & \\
\hline 1237 & 34.922 & & & 9.7 & 0.2 & & & & \\
\hline
\end{tabular}




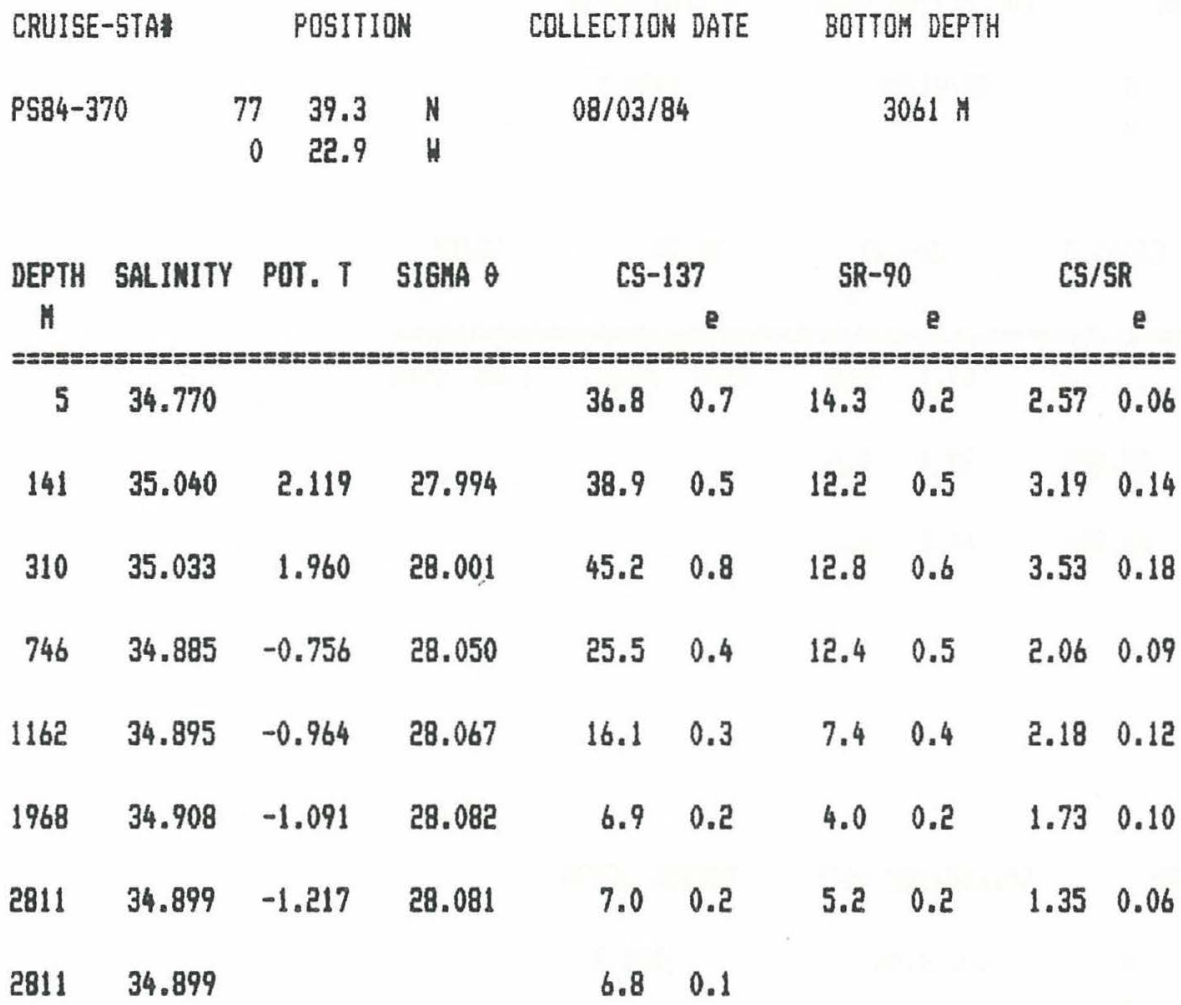

$\begin{array}{lrrrcr}\text { CRUISE-STAE } & & \text { POSITION } & \text { COLLECTION DATE } & \text { BOTTOH DEPTH } \\ \text { PS84-381 } & 77 & 40.0 & \text { N } & 08 / 04 / 84 & 3531 \text { H } \\ & 7 & 33.0 & \text { E } & & \end{array}$

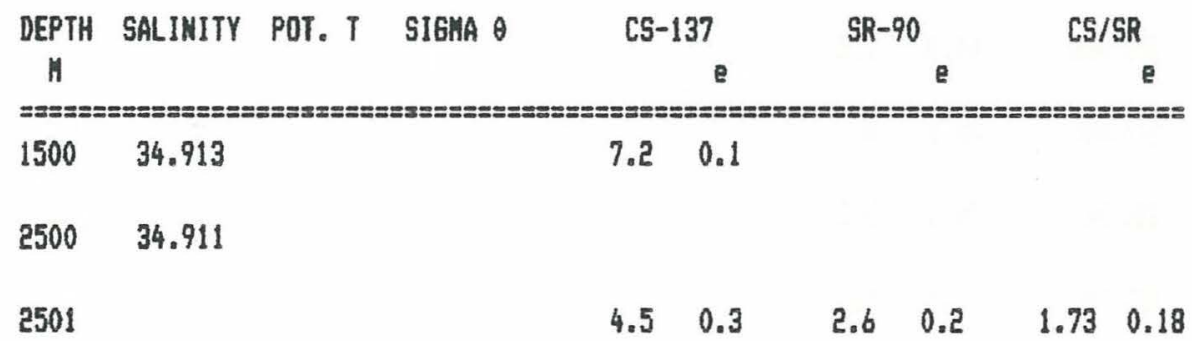

Q:1 SIGHA COUNTING UMCERTAINTY CONCENTRATIONS ARE DPM/100 KG AS OF COLLECTION DATE 


\begin{tabular}{lrr|ccr} 
CRUISE-STA\# & & POSITION & & COLLECTION DATE & BOTTOM DEPTH \\
PS84-382 & 77 & 39.9 & N & $08 / 04 / 84$ & 2153 H \\
& 8 & 29.5 & E & &
\end{tabular}

\begin{tabular}{|c|c|c|c|c|c|c|c|c|c|}
\hline \multirow{2}{*}{$\begin{array}{c}\text { DEPTH } \\
\text { H }\end{array}$} & \multirow{2}{*}{ SALINITY } & \multirow[t]{2}{*}{ POT. T } & \multirow[t]{2}{*}{ SIGHA $\theta$} & \multicolumn{2}{|c|}{ CS-137 } & \multicolumn{2}{|c|}{ SR-90 } & \multicolumn{2}{|c|}{ CS/SR } \\
\hline & & & & & e & & e & & e \\
\hline & 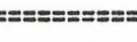 & & & $=z= \pm=$ & $= \pm=x$ & $=z==x=$ & $== \pm=$ & $==x= \pm=$ & $==x=$ \\
\hline 5 & 35.048 & & & 53.4 & 0.3 & 22.5 & 0.4 & 2.37 & 0.04 \\
\hline 46 & 35.064 & 5.281 & 27.693 & 57.0 & 0.8 & 16.2 & 0.5 & 3.52 & 0.12 \\
\hline 78 & 35.083 & 4.053 & 27.847 & 51.1 & 0.5 & 19.2 & 0.8 & 2.66 & 0.11 \\
\hline 294 & 35.061 & 2.846 & 27.948 & 46.1 & 0.3 & 17.7 & 0.8 & 2.60 & 0.12 \\
\hline 490 & 35.031 & 2.087 & 27.990 & 41.2 & 0.4 & 20.3 & 0.2 & 2.03 & 0.03 \\
\hline 739 & 34.903 & -0.081 & 28.032 & 29.6 & 0.3 & 10.8 & 0.7 & 2.74 & 0.18 \\
\hline & 34.898 & -0.643 & 28.055 & 17.4 & 0.3 & 8.8 & 0.2 & 1.98 & 0.06 \\
\hline
\end{tabular}

E:I SIGMA COUNTING UNCERTAINTY

CONCENTRATIONS ARE DPH/100 KG AS OF CQLLECTION DATE 
$-34-$ 
R/V MICHAEL SAARS: FALL 1985 CRUISE TO NORTHEAST BARENTS SEA

This cruise - courtesy of Dr. Lars Midttun of Bergen, Norway offered the opportunity to sample dense water formed on the northeast Barents Sea shelf and which is thought to contribute to shelfwater ventilation of the Arctic basins. 


\begin{tabular}{|c|c|c|c|c|c|c|c|c|c|}
\hline \multicolumn{2}{|c|}{ CRUISE-STAÜ } & \multicolumn{2}{|c|}{ POSITION } & \multicolumn{2}{|c|}{ COLLECTION DATE } & \multicolumn{4}{|c|}{ GOTTOM DEPTH } \\
\hline MSB5-A & & $\begin{array}{ll}73 & 51.0 \\
51 & 53.0\end{array}$ & $\begin{array}{l}N \\
E\end{array}$ & $09 / 00 / 8$ & & & & & \\
\hline $\begin{array}{c}\text { DEPTH } \\
\text { H }\end{array}$ & SALINITY & POT. T & & CS-1 & & SR- & & CS/ & SR \\
\hline 1 & 34.270 & & & 118.7 & 1.1 & 27.3 & 0.2 & 4.35 & 0.05 \\
\hline 237 & 34.941 & & & 116.2 & 0.8 & 24.4 & 0.3 & 4.76 & 0.07 \\
\hline
\end{tabular}

CRUISE-STA\# POSITION COLLECTION DATE BOTTOH DEPTH

$\begin{array}{lllll}\text { MS85-B } & 78 & 0.0 & N & 09 / 00 / 85\end{array}$

$\begin{array}{ccccc}\text { DEPTH SALINITY POT. T SIGMA } \theta & \text { CS- } 137 & \text { SR-90 } & \text { CS/SR } \\ & & \text { e } & \text { e } & \text { e }\end{array}$

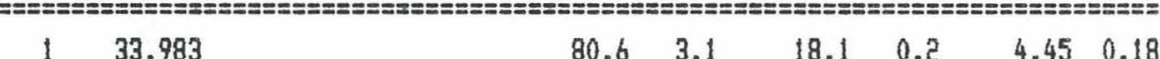

$\begin{array}{llll}260 & 34.942 & 23.3 & 0.4\end{array}$

\begin{tabular}{|c|c|c|c|c|c|}
\hline CRUISE-STAI & POSIT & & COLLECTION DATE & BOTTOH DEPTH & \\
\hline mS85-C & 30.0 & $N$ & $09 / 00 / 85$ & & \\
\hline & 0.0 & E & & & \\
\hline DEFTH SALINITY & POT. T & SIGHA $\theta$ & CS-137 & $5 R-90$ & CS/SR \\
\hline H & & & e & e & e \\
\hline & & & & & \\
\hline 134.681 & & & 111.2 & 27.9 & $\begin{array}{lll}3.78 & 0.05\end{array}$ \\
\hline
\end{tabular}

P:1 SIGHA COUNTING UNCERTAINTY

CONCENTRATIONS ARE DPH/100 KG AS OF COLLECTION DATE 


\begin{tabular}{|c|c|c|c|c|c|c|c|c|c|}
\hline \multicolumn{2}{|c|}{ CRUISE-STAA } & \multicolumn{2}{|c|}{ POSITION } & \multicolumn{2}{|c|}{ COLLECTION DATE } & \multicolumn{2}{|c|}{ BOTTOA DEFT } & & \\
\hline \multirow[t]{2}{*}{ MS85-D } & 7 & 45.0 & N & \multicolumn{2}{|c|}{$09 / 00 / 85$} & & & & \\
\hline & 5 & 0.0 & $E$ & & & & & & \\
\hline \multirow{3}{*}{ DEPTH } & \multirow[t]{2}{*}{ SALINITY } & \multirow[t]{2}{*}{ POT. T } & \multirow[t]{2}{*}{ SIGMA $\theta$} & \multicolumn{2}{|c|}{ CS-137 } & \multicolumn{2}{|c|}{$5 R-90$} & \multicolumn{2}{|c|}{ CS/SR } \\
\hline & & & & & e & & e & & e \\
\hline & 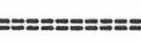 & 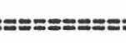 & -4 & $y==8=8= \pm=$ & :=s=sex & $:====$ & $:===$ & $:=====$ & $==x==$ \\
\hline 1 & 34.006 & & & 91.1 & 1.0 & 21.6 & 0.2 & 4.22 & 0.06 \\
\hline 285 & 34.902 & & & 45.4 & 0.4 & 22.8 & 0.3 & 1.99 & 0.03 \\
\hline
\end{tabular}

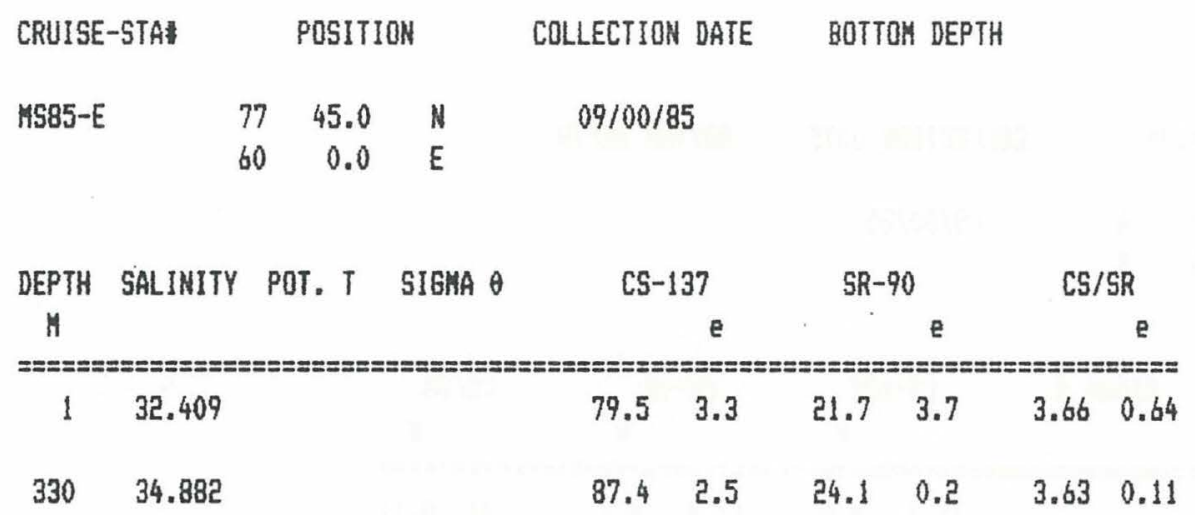

$\begin{array}{llllll}\text { CRUISE-STA } & & \text { POSITION } & \text { CQLLECTION DATE } & \text { BOTTOM DEPTH } \\ \text { MS85-F } & 75 & 21.0 & \text { N } & 09 / 00 / 85 \\ & 54 & 17.0 & \text { E } & \end{array}$

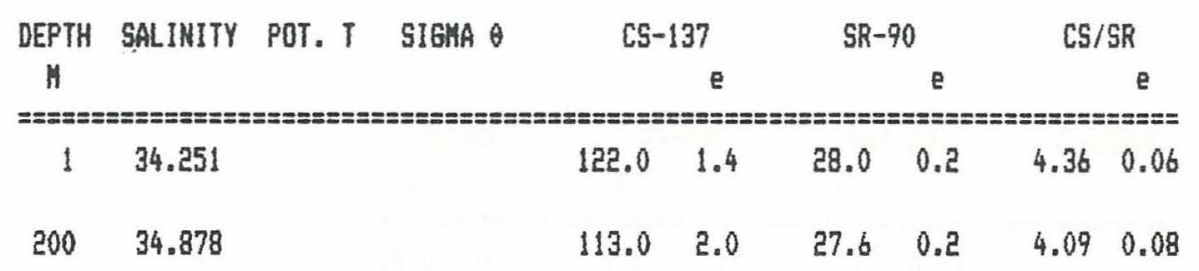




\begin{tabular}{lrrrcc} 
CRUISE-STA\# & \multicolumn{3}{c}{ POSITION } & COLLETION DATE & BOTTOH DEPTH \\
MS85-6 & 76 & 30.0 & $\mathrm{~N}$ & $09 / 00 / 85$ \\
& 50 & 0.0 & $\mathrm{E}$ &
\end{tabular}

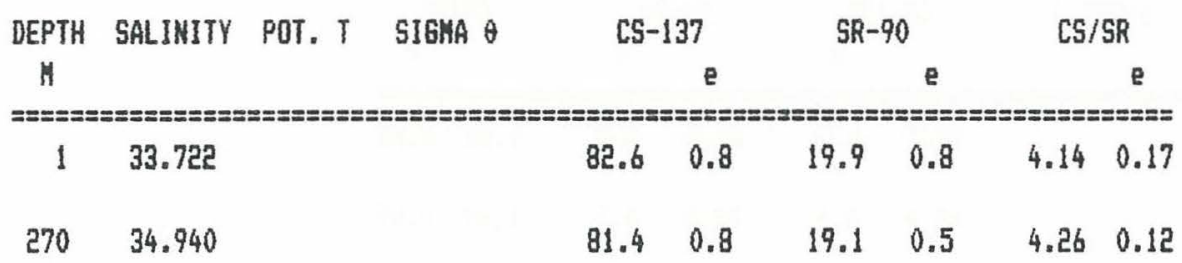

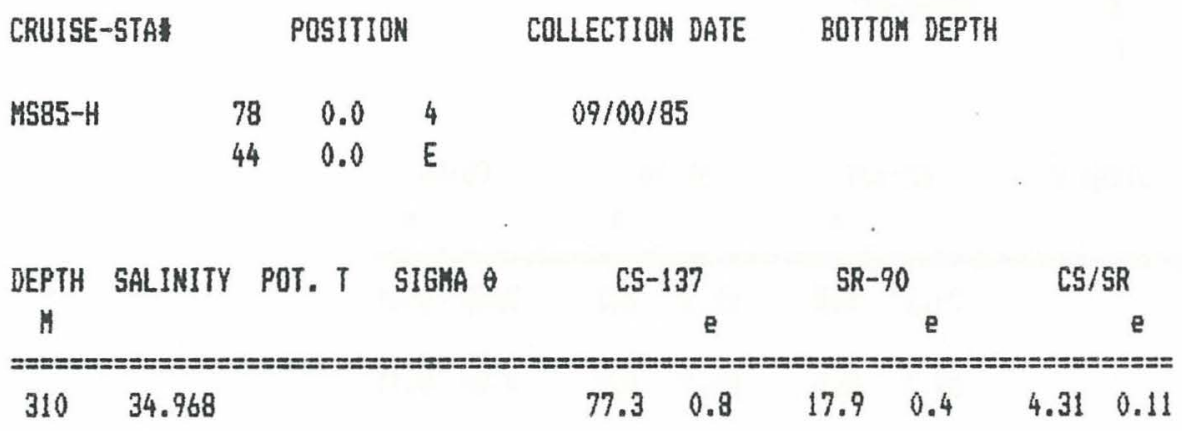

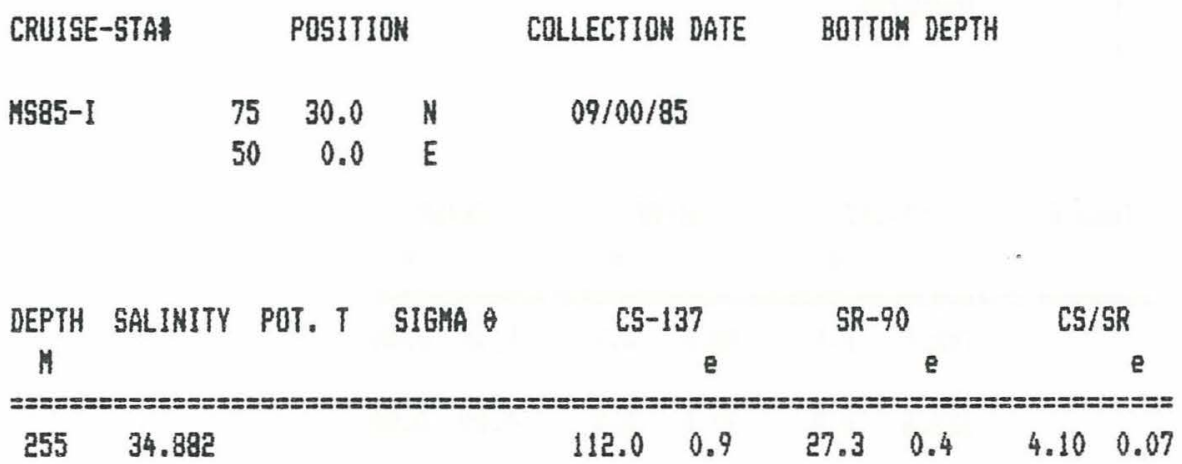




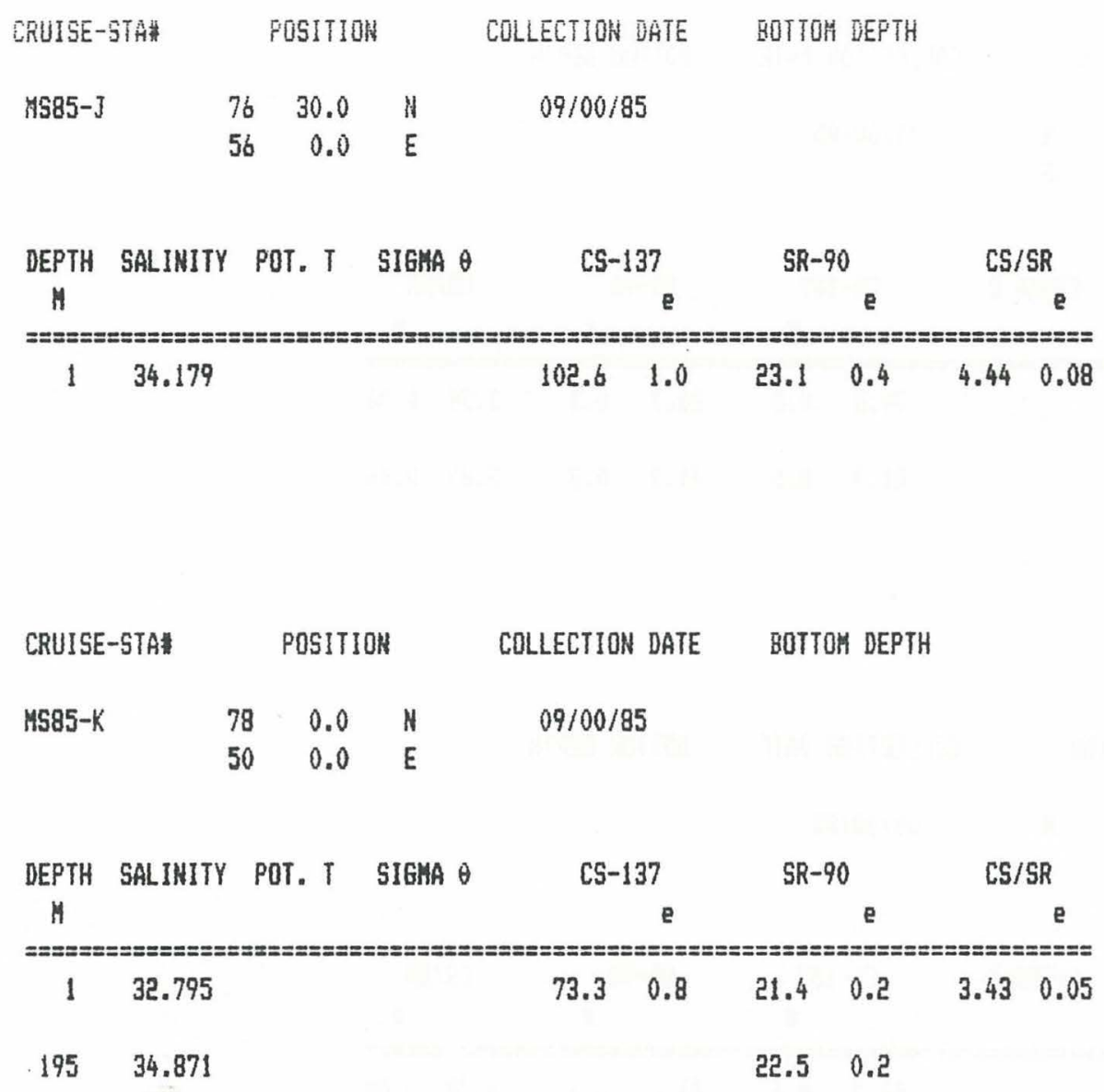

CRUISE-STA POSITION COLLECTION DATE BOTTOM DEPTH

$\begin{array}{rrrrr}M 585-L & 76 & 30.0 & N & 09 / 00 / 85\end{array}$

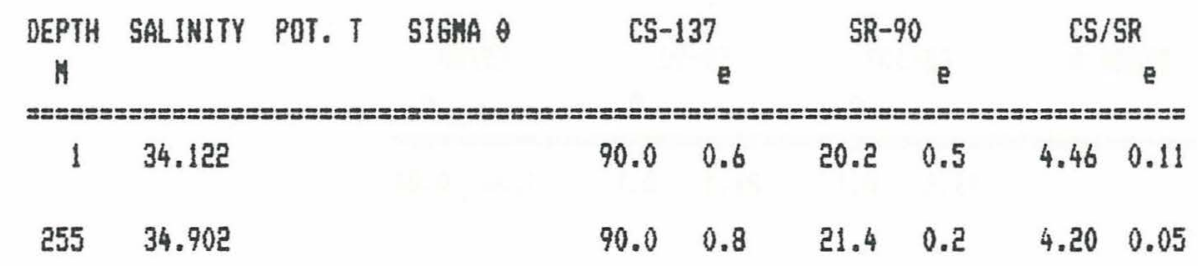

E:1 SIGMA COUNTING UNCERTAINTY CONCENTRATIONS ARE DPH/100 KG AS OF COLLECTION DATE 


\begin{tabular}{|c|c|c|c|c|c|c|c|c|c|}
\hline \multicolumn{2}{|c|}{ CRUIGE-STAZ } & \multicolumn{2}{|c|}{ POSITION } & \multicolumn{2}{|c|}{ COLLECTION DATE } & \multicolumn{4}{|c|}{ BOTTOH DEPTH } \\
\hline \multirow[t]{2}{*}{$4585-M$} & & 30.0 & N & \multicolumn{2}{|c|}{$09 / 00 / 85$} & & & & \\
\hline & & 0.0 & E & & & & & & \\
\hline \multirow{3}{*}{$\begin{array}{l}\text { DEPTH } \\
===\end{array}$} & \multirow[t]{2}{*}{ SALINITY } & \multirow[t]{2}{*}{ POT. T } & \multirow[t]{2}{*}{ SIGHA $\theta$} & \multicolumn{2}{|c|}{ CS-137 } & \multicolumn{2}{|c|}{ SR-90 } & \multicolumn{2}{|c|}{ CS/SR } \\
\hline & & & & & e & & e & & e \\
\hline & $== \pm=x= \pm=$ & ex=ex=ex= & $= \pm= \pm$ & $==x= \pm=x=x=$ & $== \pm= \pm=$ & $== \pm= \pm=$ & $== \pm=$ & $==z==$ & $:== \pm=$ \\
\hline 1 & 32.876 & & & 74.2 & 0.8 & 20.7 & 0.3 & 3.59 & 0.06 \\
\hline 220 & 34.881 & & & 81.7 & 0.5 & 21.3 & 0.3 & 3.83 & 0.06 \\
\hline
\end{tabular}

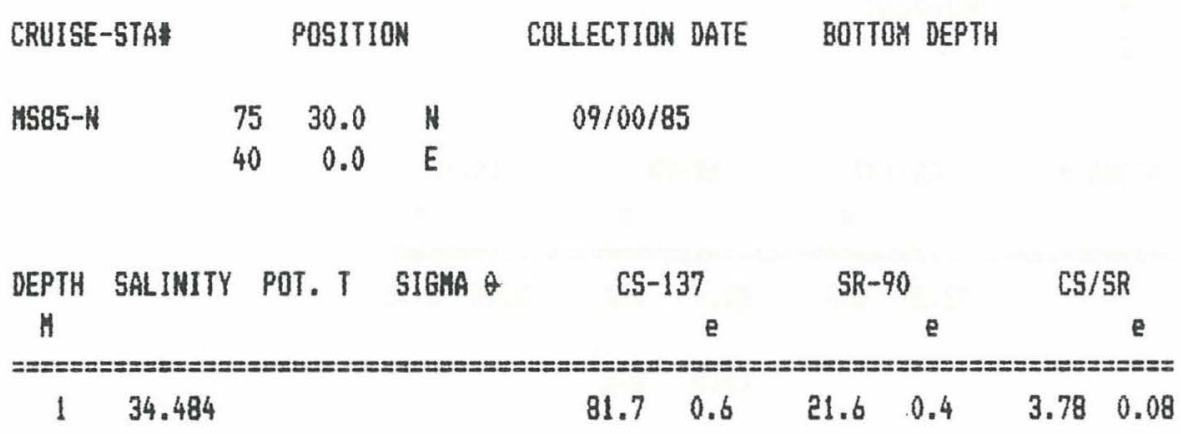

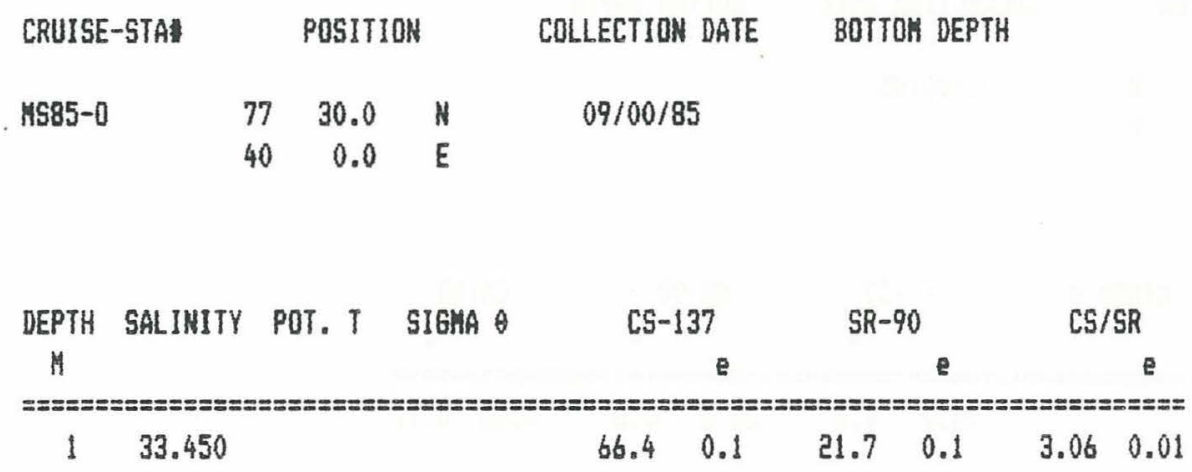

E:I SIGMA COUNTING UNCERTAINTY CONCENTRATIONS ARE DPH/100 KG AS OF COLLECTION DATE 


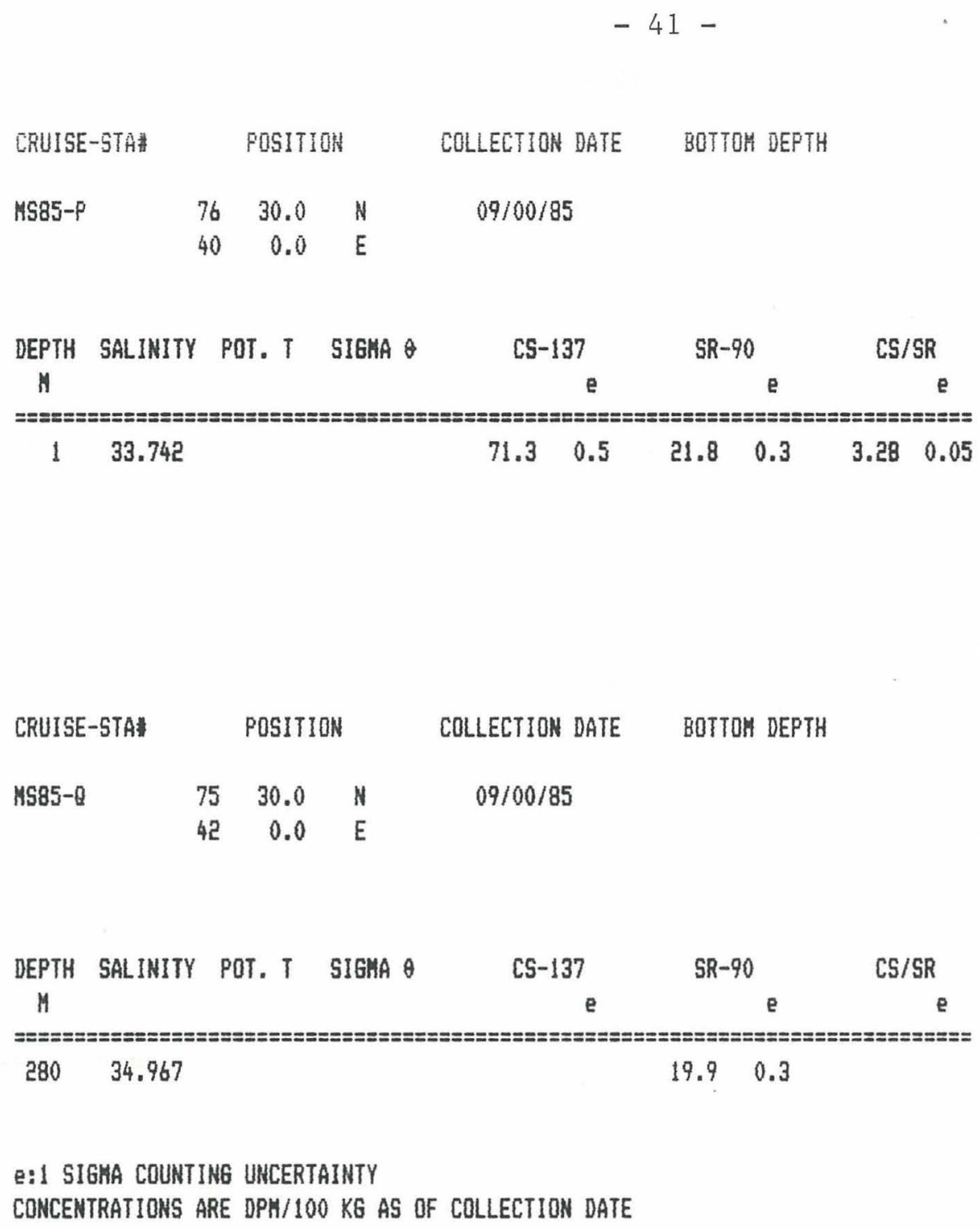


ARCTIC ICE-STATIONS: 1979-1985

This section contains data from four ice-stations occupied in 1979-1985 in the Eurasian and Canadian Basins.

1. LOREX-79: This station, on the Lomonosov Ridge, was at a location on the mid-basin ridge close to the North Pole. Deep samples were collected on both sides of the ridge.

2. FRAM-4: This camp, northeast of Fram strait on the eastern flanks of the Yermak Plateau, obtained samples of young Atlantic inflow water and deep Nansen Basin water.

3. CESAR: This station was part of the Canadian Scientific Expedition to the Alpha Ridge. Samples were collected from a part of the Canadian Basin where mixing and ventilation are relatively slow.

4. AIWEX-3: This station, north of Prudhoe Bay, was part of the Arctic International Wave Experiment. It offered the opportunity to sample water masses circulating around the margins of the Canadian Basin. 


$\begin{array}{rrrrcr}\text { CRUISE-STA\# } & & \text { POSITION } & \text { COLLECTION DATE } & \text { BOTTOH DEFTH } \\ \text { LOREX-79 } & 89 & 0.0 & \mathrm{~N} & 04 / 00 / 79 & 3000 \mathrm{M} \\ & 120 & 0.0 & \mathrm{H} & & \end{array}$

POSITION (LONG.) IS AVE.; SEE MANUSCRIPT

\begin{tabular}{|c|c|c|c|c|c|c|c|c|c|c|c|}
\hline \multirow{2}{*}{ DEPTH } & \multirow[t]{2}{*}{ SALINITY } & \multirow[t]{2}{*}{ POT. T } & \multirow[t]{2}{*}{ SIGHA $\theta$} & \multicolumn{2}{|c|}{$\operatorname{cs} 137$} & \multicolumn{2}{|c|}{ SR90 } & \multicolumn{2}{|c|}{ PUe39 } & \multicolumn{2}{|c|}{ AM241 } \\
\hline & & & & \multicolumn{2}{|c|}{ e } & \multirow{2}{*}{\multicolumn{2}{|c|}{ 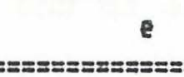 }} & \multicolumn{2}{|c|}{ e } & \multicolumn{2}{|c|}{ e } \\
\hline \multicolumn{10}{|c|}{ 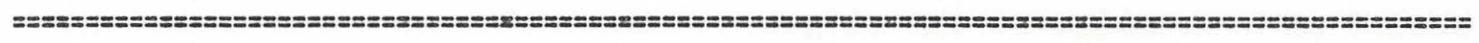 } & & \\
\hline 2 & 30.000 & -1.600 & & 39.2 & 0.5 & 31.6 & 0.7 & 0.082 & 0.013 & 0.042 & 0.011 \\
\hline 75 & 32.000 & -1.600 & & 33.6 & 0.4 & 33.1 & 4.4 & 0.098 & 0.012 & 0.031 & 0.007 \\
\hline 110 & 33.500 & -1.500 & & 25.5 & 0.5 & 25.5 & 1.0 & 0.045 & 0.008 & -.005 & 0.007 \\
\hline 500 & 34.880 & 0.510 & & 7.3 & 0.4 & 4.5 & 0.1 & 0.110 & 0.010 & 0.025 & 0.006 \\
\hline 1000 & 34.901 & -0.170 & & 6.6 & 0.3 & 3.8 & 0.3 & 0.061 & 0.014 & 0.019 & 0.005 \\
\hline 1500 & 34.920 & -0.520 & & 18.5 & 0.4 & 8.7 & 0.1 & 0.068 & 0.007 & 0.018 & 0.005 \\
\hline 2497 & 34.940 & -0.850 & & 7.3 & 0.2 & 4.8 & 0.7 & 0.035 & 0.006 & 0.003 & 0.002 \\
\hline 3000 & 34.953 & -0.460 & & 0.9 & 0.3 & 0.6 & 0.3 & 0.012 & 0.005 & 0.004 & 0.002 \\
\hline
\end{tabular}

P:1 SIGMA COUNTING UNCERTAINTY

CONCENTRATIONS ARE DPM/100 KG AS OF COLLECTION DATE 


$\begin{array}{llllcc}\text { CRUISE-STA\# } & & \text { POSITION } & \text { COLLECTION DATE } & \text { BOTTOM DEPTH } \\ \text { FRAM-4 } & 83 & 33.0 & \text { N } & 04 / 15 / 82 & 3800 ~ \\ & 15 & 17.0 & \text { E } & & \end{array}$

SALINITY MAX AT $230 \mathrm{H}$

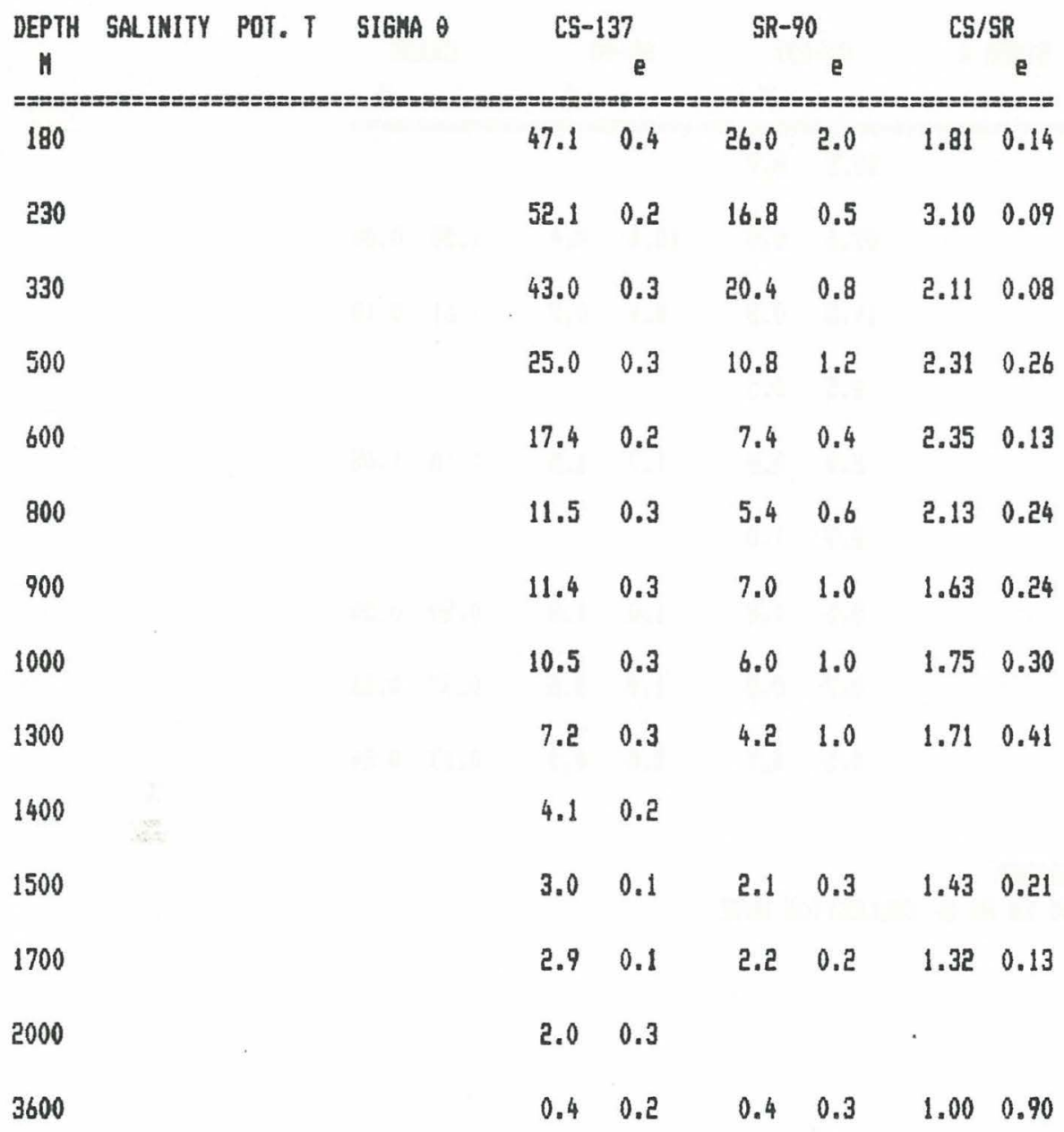

Q:1 SIGMA COUNTING UNCERTAINTY

CONCENTRATIONS ARE DPM/100 KG AS OF COLLECTION DATE 


\begin{tabular}{|c|c|c|c|c|c|c|c|c|c|}
\hline \multicolumn{2}{|c|}{ CRUISE-STA\# } & \multicolumn{2}{|c|}{ POSITION } & \multirow{2}{*}{\multicolumn{2}{|c|}{$\begin{array}{l}\text { COLLECTION DATE } \\
\text { 04/15/83 }\end{array}$}} & \multicolumn{4}{|c|}{ BOTTOH DEPTH } \\
\hline CESAR & $\begin{array}{r}8 \\
13 x\end{array}$ & $\begin{array}{l}0.0 \\
0.0\end{array}$ & $\begin{array}{l}N \\
H\end{array}$ & & & & & & \\
\hline \multicolumn{10}{|c|}{ TEMP NOT POT. TEMP } \\
\hline \multirow{2}{*}{$\begin{array}{l}\text { DEPTH } \\
=== \pm=\end{array}$} & SALINITY & POT. T & SIGHA $\theta$ & Cs-1 & & SR-9 & & $\mathrm{CS} / \mathrm{s}$ & $\begin{array}{l}\text { SR } \\
\text { e }\end{array}$ \\
\hline & 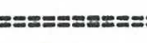 & $== \pm= \pm==x=$ & $== \pm= \pm=x=2$ & 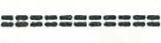 & $==x== \pm=8$ & $=z== \pm=$ & $z==2= \pm=2$ & $== \pm==2$ & $== \pm==$ \\
\hline 2 & 31.831 & & & 31.5 & 0.9 & & & & \\
\hline 172 & 34.116 & -1.040 & & 27.6 & 0.4 & 18.4 & 0.4 & 1.50 & 0.04 \\
\hline 240 & 34.613 & & & 14.3 & 0.3 & 8.9 & 0.7 & 1.61 & 0.13 \\
\hline 602 & 34.891 & & & 2.5 & 0.5 & & & & \\
\hline 800 & 34.901 & -0.035 & & 2.0 & 0.2 & 1.7 & 1.5 & 1.18 & 1.05 \\
\hline 1000 & 34.917 & -0.090 & & 2.7 & 1.0 & & & & \\
\hline 1300 & 34.937 & & & 0.5 & 0.2 & 1.0 & 1.0 & 0.50 & 0.54 \\
\hline 1400 & 34.938 & & & 0.7 & 0.2 & 1.9 & 0.6 & 0.37 & 0.16 \\
\hline 1500 & 34.934 & -0.250 & & 0.5 & 0.7 & 3.0 & 0.0 & 0.17 & 0.24 \\
\hline
\end{tabular}




\begin{tabular}{|c|c|c|c|c|c|c|c|c|c|}
\hline \multicolumn{2}{|c|}{ CRUISE-STAE } & \multicolumn{2}{|c|}{ POSITION } & \multicolumn{2}{|c|}{ COLLECTION DATE } & \multicolumn{2}{|c|}{ BOTTOH DEPTH } & & \\
\hline \multirow{2}{*}{\multicolumn{2}{|c|}{ AIHEX -3}} & 8.4 & N & \multirow{2}{*}{\multicolumn{2}{|c|}{$04 / 08 / 85$}} & \multirow{2}{*}{\multicolumn{2}{|c|}{$3707 \mathrm{M}$}} & & \\
\hline & & 39.2 & $H$ & & & & & & \\
\hline DEPTH & SALINITY & POT. T & SIGMA $\theta$ & \multicolumn{2}{|c|}{ CS-137 } & \multicolumn{2}{|c|}{ SR-90 } & \multicolumn{2}{|c|}{ CS/SR } \\
\hline H & & & & & e & & e & & e \\
\hline \multicolumn{10}{|c|}{ 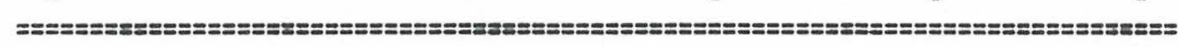 } \\
\hline 5 & 30.286 & -1.420 & 24.343 & 45.4 & 0.3 & 32.6 & 0.3 & 1.39 & 0.02 \\
\hline 155 & 32.911 & -1.460 & 26.474 & 27.0 & 0.3 & 18.2 & 0.6 & 1.48 & 0.05 \\
\hline 197 & 33.319 & -1.500 & 26.807 & 23.7 & 0.3 & 16.2 & 0.3 & 1.46 & 0.03 \\
\hline 237 & 33.922 & -1.100 & 27.283 & 13.3 & 0.2 & 8.0 & 0.8 & 1.66 & 0.17 \\
\hline 388 & 34.795 & 0.384 & 27.919 & 5.6 & 0.2 & 2.5 & 0.2 & 2.24 & 0.20 \\
\hline 506 & 34.859 & 0.450 & 27.967 & 4.6 & 0.2 & 2.2 & 0.3 & 2.09 & 0.30 \\
\hline 725 & 34.891 & 0.226 & 28.006 & 7.5 & 0.2 & 3.0 & 0.4 & 2.50 & 0.34 \\
\hline 843 & 34.895 & 0.072 & 28.017 & 6.9 & 0.2 & 4.5 & 0.2 & 1.53 & 0.08 \\
\hline 1233 & 34.913 & -0.275 & 28.050 & 8.0 & 0.3 & 3.0 & 0.4 & 2.67 & 0.37 \\
\hline 1931 & 34.942 & -0.516 & 28.085 & 2.4 & 0.3 & 1.5 & 0.3 & 1.60 & 0.38 \\
\hline 3663 & 34.953 & -0.514 & 28.094 & 0.2 & 0.2 & 0.3 & 1.0 & 0.67 & 2.33 \\
\hline
\end{tabular}




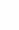


Attn: Stella Sanchez-Wade

Documents Section

Scripps Institution of Oceanography

Library, Mail Code C-075C

La Jolla, CA 92093

Hancock Library of Biology \& Oceanography

Alan Hancock Laboratory

University of Southern California University Park

Los Angeles, CA 90089-0371

Gifts \& Exchanges

Library

Bedford Institute of Oceanography

P.O. Box 1006

Dartmouth, NS, B2Y 4A2, CANADA

Office of the International Ice Patrol

c/o Coast Guard R \& D Center

Avery Point

Groton, CT 06340

Library

Physical Oceanographic Laboratory

Nova University

8000 N. Ocean Drive

Dania, FL 33304

NOAA/EDIS Miami Library Center 4301 Rickenbacker Causeway

Miami, FL 33149

Library

Skidaway Institute of Oceanography

P.O. Box 13687

Savannah, GA 31416

Institute of Geophysics

University of Hawaii

Library Room 252

2525 Correa Road

Honolulu, HI 96822

Library

Chesapeake Bay Institute

4800 Atwell Road

Shady Side, MD 20876

MIT Libraries

Serial Journal Room 14E-210

Cambridge, MA 02139
Director, Ralph M. Parsons Laboratory

Room 48-31 1

MIT

Cambridge, MA 02139

Marine Resources Information Center

Building E38-320

MIT

Cambridge, MA 02139

Library

Lamont-Doherty Geological Observatory

Colombia University

Palisades, NY 10964

Library

Serials Department

Oregon State University

Corvallis, OR 97331

Pell Marine Science Library

University of Rhode Island

Narragansett Bay Campus

Narragansett, RI 02882

Working Collection

Texas A\&M University

Dept. of Oceanography

College Station, TX 77843

Library

Virginia Institute of Marine Science

Gloucester Point, VA 23062

Fisheries-Oceanography Library

151 Oceanography Teaching Bldg.

University of Washington

Seattle, WA 98195

Library

R.S.M.A.S.

University of Miami

4600 Rickenbacker Causeway

Miami, FL 33149

Maury Oceanographic Library

Naval Oceanographic Office

Bay St. Louis

NSTL, MS 39522-5001 



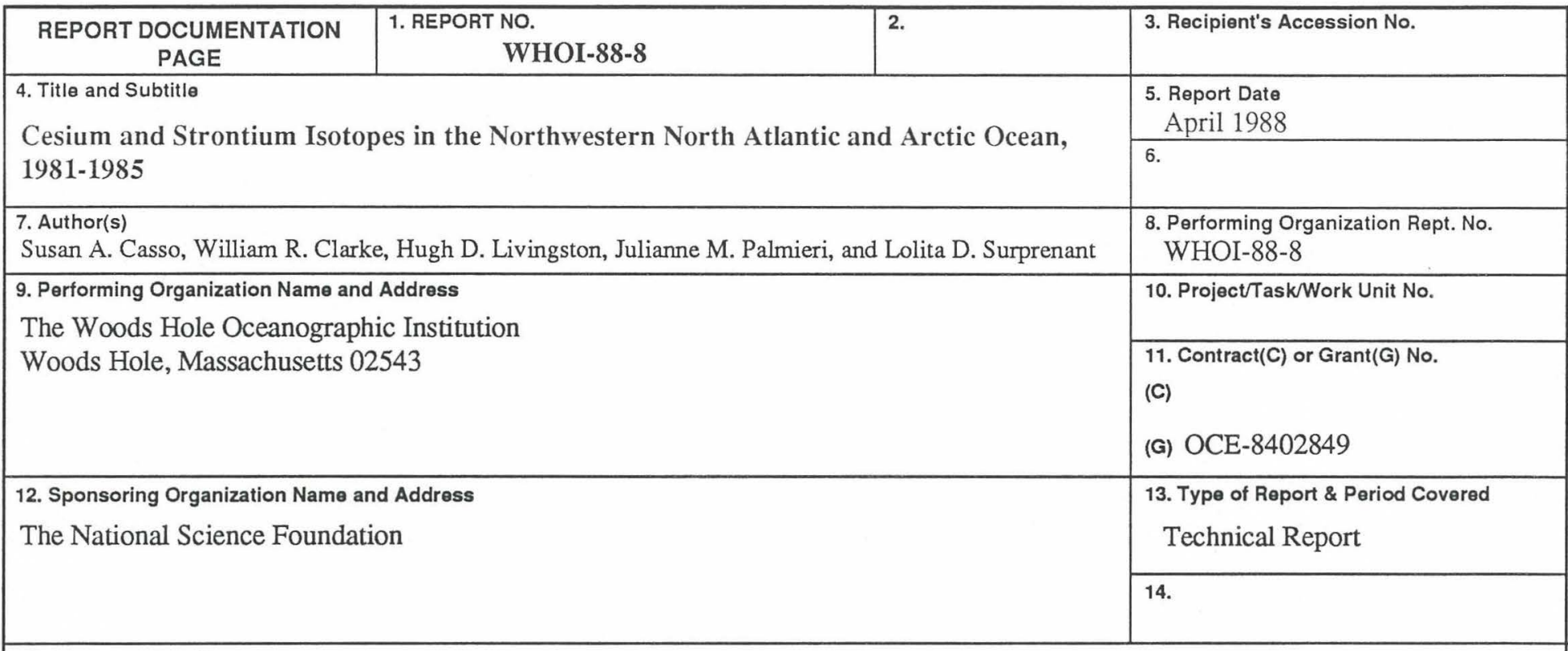

\section{Supplementary Notes}

This report should be cited as: Woods Hole Oceanog. Inst. Tech. Rept., WHOI-88-8.

16. Abstract (Limit: 200 words)

This report is a follow-up to the Woods Hole Oceanographic Institution Technical Report WHOI-84-40. It contains ${ }^{137} \mathrm{Cs}$ and ${ }^{90} \mathrm{Sr}$ data from seawater samples collected on four cruises in the northwestern North Atlantic, Arctic Ocean, and Barents Sea during 1981 to 1985 , and radionuclide data $\left({ }^{137} \mathrm{Cs},{ }^{90} \mathrm{Sr}\right.$ and $\left.{ }^{239} \mathrm{Pu}\right)$ from samples collected on a cruise to the Norwegian-Greenland Seas in 1979 . Also included are data from four ice stations in the Arctic from 1979 to 1985 . The sample collections were made possible through collaborative efforts with several laboratories. The radionuclide analyses were done at the Woods Hole Oceanographic Institution.

17. Document Analysis a. Descriptors

1. Cs-137

2. Sr-90

3. Arctic Ocean

b. Identifiers/Open-Ended Terms

c. COSATI Field/Group

18. Availability Statement

Approved for publication; distribution unlimited.

\begin{tabular}{|c|c|}
\hline $\begin{array}{c}\text { 19. Security Class (This Report) } \\
\text { UNCLASSIFIED }\end{array}$ & $\begin{array}{c}\text { 21. No. of Pages } \\
47\end{array}$ \\
\hline 20. Security Class (This Page) & 22. Price \\
\hline
\end{tabular}


